\title{
The Murchison Widefield Array: The Square Kilometre Array Precursor at Low Radio Frequencies
}

\author{
S. J. Tingay ${ }^{1,2,20}$, R. Goeke ${ }^{3}$, J. D. Bowman ${ }^{4}$, D. Emrich $^{1}$, S. M. Ord ${ }^{1}$, D. A. Mitchell2,5, M. F. Morales ${ }^{6}$, \\ T. Booler ${ }^{1}$, B. Crosse ${ }^{1}$, R. B. Wayth ${ }^{1,2}$, C. J. Lonsdale ${ }^{7}$, S. Tremblay ${ }^{1,2}$, D. Pallot ${ }^{1}$, T. Colegate ${ }^{1}$, \\ A. Wicenec ${ }^{8}$, N. Kudryavtseva ${ }^{1}$, W. Arcus ${ }^{1}$, D. Barnes ${ }^{9}$, G. Bernardi ${ }^{10}$, F. Briggs ${ }^{2,11}$, S. Burns ${ }^{12}$, \\ J. D. Bunton ${ }^{13}$, R. J. Cappallo ${ }^{7}$, B. E. Corey ${ }^{7}$, A. Deshpande ${ }^{14}$, L. Desouza ${ }^{13}$, B. M. Gaensler ${ }^{2,15}$, \\ L. J. Greenhill ${ }^{10}$, P. J. Hall ${ }^{1}$, B. J. Hazelton ${ }^{6}$, D. Herne ${ }^{1}$, J. N. Hewitt ${ }^{3}$, M. Johnston-Hollitt ${ }^{16}$, \\ D. L. Kaplan ${ }^{17}$, J. C. Kasper ${ }^{10}$, B. B. Kincaid ${ }^{7}$, R. Koenig ${ }^{13}$, E. Kratzenberg ${ }^{7}$, M. J. Lynch ${ }^{1}$, \\ B. Mckinley ${ }^{2,11}$, S. R. Mcwhirter ${ }^{7}$, E. Morgan ${ }^{3}$, D. Oberoi ${ }^{18}$, J. Pathikulangara ${ }^{13}$, T. Prabu ${ }^{14}$, \\ R. A. Remillard ${ }^{3}$, A. E. E. Rogers ${ }^{7}$, A. Roshi ${ }^{19}$, J. E. Salah ${ }^{7}$, R. J. Sault ${ }^{5}$, N. Udaya-Shankar ${ }^{14}$, \\ F. Schlagenhaufer ${ }^{1}$, K. S. Srivani ${ }^{14}$, J. Stevens ${ }^{13}$, R. Subrahmanyan ${ }^{2,14}$, M. Waterson ${ }^{1}$, R. L. Webster ${ }^{2,5}$, \\ A. R. Whitney ${ }^{7}$, A. Williams ${ }^{8}$, C. L. Williams ${ }^{3}$ and J. S. B. Wyithe ${ }^{2,5}$ \\ ${ }^{1}$ ICRAR - Curtin University, Perth, Australia \\ ${ }^{2}$ ARC Centre of Excellence for All-sky Astrophysics (CAASTRO), Redfern, NSW, Australia \\ ${ }^{3}$ MIT Kavli Institute for Astrophysics and Space Research, Cambridge, MA, USA \\ ${ }^{4}$ School of Earth and Space Exploration, Arizona State University, Tempe, AZ, USA \\ ${ }^{5}$ School of Physics, The University of Melbourne, Melbourne, Australia \\ ${ }^{6}$ Physics Department, University of Washington, Seattle, WA, USA \\ ${ }^{7}$ MIT Haystack Observatory, Westford, MA, USA \\ ${ }^{8}$ ICRAR - University of Western Australia, Perth, Australia \\ ${ }^{9}$ Monash e-Research Centre, Monash University, Melbourne, Australia \\ ${ }^{10}$ Harvard-Smithsonian Center for Astrophysics, Cambridge, MA, USA \\ ${ }^{11}$ Research School of Astronomy and Astrophysics, The Australian National University, Canberra, Australia \\ ${ }^{12}$ Burns Industries, Nashua, $\mathrm{NH}$, USA \\ ${ }^{13}$ CSIRO Astronomy and Space Science, Australia \\ ${ }^{14}$ Raman Research Institute, Bangalore, India \\ ${ }^{15}$ Sydney Institute for Astronomy, The University of Sydney, Sydney, Australia \\ ${ }^{16}$ School of Chemical and Physical Sciences, Victoria University of Wellington, Wellington, New Zealand \\ ${ }^{17}$ Physics Department, University of Wisconsin-Milwaukee, Milwaukee, WI, USA \\ ${ }^{18}$ National Centre for Radio Astrophysics, Pune, India \\ ${ }^{19}$ National Radio Astronomy Observatory, Charlottesville, WV, USA \\ ${ }^{20}$ Email: s.tingay@ curtin.edu.au
}

(Received June 22, 2012; Accepted September 1, 2012; OnLine Publication January 24, 2013)

\begin{abstract}
The Murchison Widefield Array (MWA) is one of three Square Kilometre Array Precursor telescopes and is located at the Murchison Radio-astronomy Observatory in the Murchison Shire of the mid-west of Western Australia, a location chosen for its extremely low levels of radio frequency interference. The MWA operates at low radio frequencies, 80$300 \mathrm{MHz}$, with a processed bandwidth of $30.72 \mathrm{MHz}$ for both linear polarisations, and consists of 128 aperture arrays (known as tiles) distributed over a $\sim 3-\mathrm{km}$ diameter area. Novel hybrid hardware/software correlation and a real-time imaging and calibration systems comprise the MWA signal processing backend. In this paper, the as-built MWA is described both at a system and sub-system level, the expected performance of the array is presented, and the science goals of the instrument are summarised.
\end{abstract}

Keywords: instrumentation: interferometers, techniques: image processing, techniques: interferometric — radio continuum: general, radio lines: general, early Universe 


\section{INTRODUCTION}

The Murchison Widefield Array ${ }^{1}$ (MWA) is the Square Kilometre Array (SKA; Dewdney et al. 2010) Precursor telescope at low radio frequencies. An SKA Precursor is a recognised SKA science and technology demonstrator located at one of the two sites shortlisted for the SKA in 2006, the Murchison Radio-astronomy Observatory (MRO) in the Murchison region of Western Australia and the Karoo region of South Africa's Northern Cape. ${ }^{2}$ The MWA is one of the two SKA Precursor telescopes sited at the MRO, the other being the Australian SKA Pathfinder (ASKAP; Johnston et al. 2007, 2008). The MeerKAT ${ }^{3}$ SKA Precursor is located at the South African site. The MRO was chosen for the site of the MWA due to its extremely low levels of human-made radio frequency interference, particularly in the FM band encompassed by the MWA at the low end of its operating frequency range (Bowman \& Rogers 2010). The MRO has been chosen as the host site for the low-frequency component of the SKA, in both Phases 1 and 2, to consist of sparse aperture arrays. ${ }^{4}$

While only three instruments have SKA Precursor status, a number of other instruments have SKA Pathfinder status (SKA technology demonstrator but not on a candidate SKA site) and are also feeding information into the SKA design and costing process. The most notable of the SKA Pathfinders in the MWA frequency range is Low Frequency Array, built in the Netherlands (M. van Harlem et al., in preparation).

The MWA and ASKAP are complementary, in that they operate in different frequency ranges (MWA: $80-300 \mathrm{MHz}$; ASKAP: $0.7-1.8 \mathrm{GHz}$ ) and employ different antenna technologies [MWA: aperture arrays (tiles); ASKAP: dishes plus Phased Array Feeds]. The combined MWA and ASKAP technology specifications almost fully sample the road map technologies for the SKA (Dewdney et al. 2010) at a single radio-quiet location, the MRO. In particular, the MWA explores the so-called large- $\mathrm{N}$ and small-D array concept that will be utilised for the SKA, with large numbers of small receiving elements providing a large field of view (FoV) on the sky and therefore high sensitivity over wide fields, equating to high survey speed, a key metric for SKA science (Carilli \& Rawlings 2004).

All three of the MWA, ASKAP and MeerKAT are planning, or have operated, demonstrator instruments. ASKAP is building the six-antenna Boolardy Engineering Test Array ${ }^{5}$ (BETA). MeerKAT is being preceded by KAT7, ${ }^{6}$ a sevenantenna array. For the MWA, a 32-tile test array operated between 2009 and 2011, allowing the assessment of a number of generations of prototype hardware as well as several iter-

\footnotetext{
${ }^{1}$ http://www.mwatelescope.org; http://www.facebook.com/Murchison. Widefield.Array

2 The term 'SKA Precursor' does not imply that the design decisions made for the MWA will be adopted for the SKA.

${ }^{3} \mathrm{http}: / /$ public.ska.ac.za/meerkat

${ }^{4}$ http://www.skatelescope.org/news/dual-site-agreed-square-kilometrearray-telescope/

${ }^{5} \mathrm{http}: / /$ www.atnf.csiro.au/projects/askap

${ }^{6} \mathrm{http}: / /$ public.ska.ac.za/kat-7
}

ations of MWA system integration. The MWA 32-tile array also undertook science-quality astronomical observations, in order to demonstrate the performance of the hardware onsky. A number of science results have been reported from the 32-tile data (Oberoi et al. 2011; Williams et al. 2012; M. Bell et al., in preparation; G. Bernardi et al. 2012; B. McKinley et al. 2012). The operation of the 32-tile test array ceased in late 2011, in preparation for the commencement of construction for the final MWA instrument in early 2012, due for completion in late 2012.

Previously, Lonsdale et al. (2009) described the conceptual underpinnings of the MWA design, the advantages of a large-N, small-D architecture for radio interferometers, and some of the challenges inherent in the data processing for this type of telescope. Lonsdale et al. (2009) also provided brief descriptions of the initial plans for MWA hardware and data processing elements, as well as the science goals for the MWA.

Since the Lonsdale et al. (2009) paper was published, a number of design and construction considerations, which were uncertain at the time of publication, have been finalised. In particular, the originally envisaged 512 tiles were re-scoped to 128 tiles due to funding constraints. The construction of the final MWA instrument is underway and practical completion of the facility is expected in late 2012. MWA commissioning commenced in mid-2012, with science operations to commence in approximately mid-2013. Thus, the purpose of this paper is to provide a full description of the MWA in its as-built form, including the final system architecture and sub-systems, as well as signal processing and data handling strategies (Section 2). This paper will also give a brief summary of the MWA science goals, a full description of which will appear elsewhere (J. Bowman et al. 2012), and details on the expected performance of the MWA (Sections 3 and 4). This paper is intended to inform future users of the capabilities of the MWA, ahead of the science operations phase, such that users can commence planning for MWA science activities. Section 5 briefly discusses the MWA within the context of other existing and future instrumentation, including the Phase 1 SKA.

\section{MWA SYSTEM DESIGN AND SUB-SYSTEM DESCRIPTIONS}

We start with a very brief overview of the MWA signal path and provide details for each of the identified subsystems in subsequent sections. Table 1 provides a summary of the MWA system parameters and expected performance. Figure 1 gives a high-level schematic overview of the MWA physical system and signal path.

The MWA signal path starts with a dual-polarisation dipole antenna, roughly a square metre of collecting area at $\sim 150$ $\mathrm{MHz}$. Sixteen of these antennas are configured as an aperture array on a regular $4 \times 4$ grid (with a spacing of $1.1 \mathrm{~m}$ ). Their signals are combined in an analog beamformer, using a set of switchable delay lines to provide coarse pointing 
Table 1. System Parameters for the MWA

\begin{tabular}{|c|c|c|c|}
\hline Parameter & Symbol & $150 \mathrm{MHz}$ & $200 \mathrm{MHz}$ \\
\hline Number of tiles & $N$ & 128 & 128 \\
\hline Area of one tile at zenith $\left(\mathrm{m}^{2}\right)$ & $A_{\text {eff }}$ & 21.5 & 19.8 \\
\hline Total collecting area $\left(\mathrm{m}^{2}\right)$ & & 2752 & 2534 \\
\hline Receiver temperature $(\mathrm{K})$ & $T_{\text {rcv }}$ & 50 & 25 \\
\hline Typical sky temperature $^{a}(\mathrm{~K})$ & $T_{\text {sky }}$ & 350 & 170 \\
\hline Field of view ${ }^{b}\left(\mathrm{deg}^{2}\right)$ & $\Omega_{\mathrm{P}}^{\text {sky }}$ & 610 & 375 \\
\hline Instantaneous bandwidth (MHz) & ${ }_{B}^{P}$ & 30.72 & 30.72 \\
\hline Spectral resolution (MHz) & & 0.04 & 0.04 \\
\hline Temporal resolution & & $\begin{array}{c}0.5 \mathrm{~s} \text { uncalibrated } \\
8 \mathrm{~s} \text { calibrated }\end{array}$ & $\begin{array}{c}0.5 \mathrm{~s} \text { uncalibrated } \\
8 \mathrm{~s} \text { calibrated }\end{array}$ \\
\hline Polarisation & & Full Stokes & Full Stokes \\
\hline Minimum baseline $(\mathrm{m})$ & & 7.7 & 7.7 \\
\hline Maximum baseline (m) & & 2864 & 2864 \\
\hline Angular resolution (1.5-km array) & & $\sim 3 \operatorname{arcmin}$ & $\sim 2 \operatorname{arcmin}$ \\
\hline Angular resolution (3-km array) & & $\sim 2 \operatorname{arcmin}$ & $\sim 1 \operatorname{arcmin}$ \\
\hline
\end{tabular}

${ }^{a}$ Nijboer, Pandey-Pommier, \& de Bruyn (2009).

${ }^{b}$ Based on the FWHM of the primary beam. Imageable area is significantly larger.

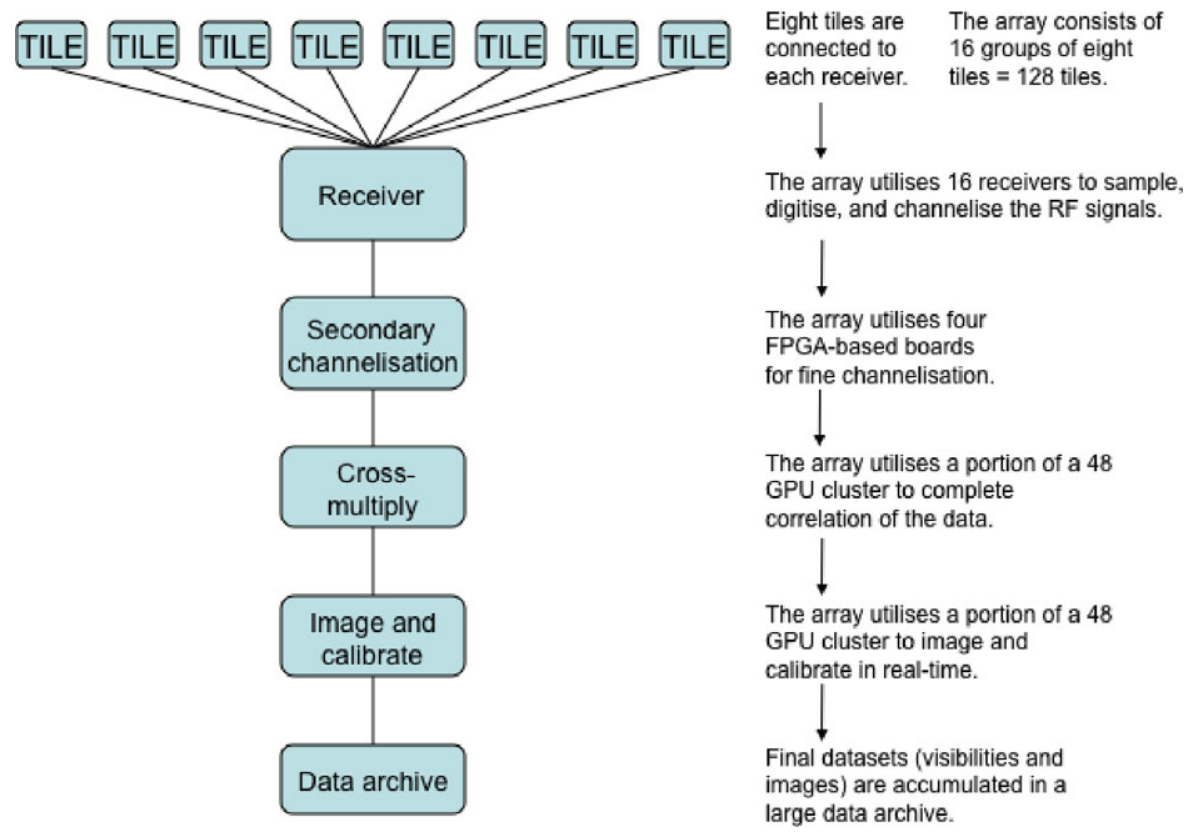

Figure 1. High-level schematic overview of the MWA physical system and signal path.

capability. Each beamformer produces two wideband analog outputs representing orthogonal $\mathrm{X}$ and $\mathrm{Y}$ linear polarisations. This we refer to as an antenna tile and analog beamformer (Section 2.3).

The sum of our four science drivers (Section 4 and J. Bowman et al. 2012) leads to a desire for a $u v$ baseline distribution which has a dense core surrounded by a smooth $r^{-2}$ radial distribution (Section 2.2). Our core area has 50 tiles uniformly distributed over a 100-m diameter core, surrounded by 62 tiles which are distributed over a $1.5-\mathrm{km}$ diameter circle. The final 16 tiles have been placed even further out on a 3-km diameter circle to optimise solar imaging performance, and for the highest angular resolution imaging.
A host of practical considerations led us to combine the signals from eight tiles into a single receiver (Section 2.4); we thus deploy 16 receivers distributed over our landscape such that no tile is more than $500 \mathrm{~m}$ removed from its associated receiver. A receiver filters the two analog signals from each tile to a bandpass of 80-300 MHz, Nyquist samples the signals with an 8-bit $\mathrm{A} / \mathrm{D}$ converter and digitally filters the result into $24 \times 1.28 \mathrm{MHz}$ frequency channels which form a selectable, usually but not necessarily contiguous, 30.72$\mathrm{MHz}$ sample space. The receivers themselves are a mixture of high technology and low, combining high-speed, and hence high power, digital circuitry with mechanical cooling. They are powered from a standard $240-\mathrm{V}$ mains circuit and send 
out their digital data streams on three 2.1-Gbps fibre optic links. One of our early design decisions was to provide spare power and fibre connections to most receiver locations so that future instrument development could share our existing infrastructure at low incremental cost.

About $5 \mathrm{~km}$ from the core of our array sits a building provided by the Commonwealth Scientific and Industrial Research Organisation (CSIRO), which we share with the ASKAP project (the Central Processing Facility, CPF). It has power, electromagnetic interference (EMI) shielding, and water-cooled equipment racks for our data processing hardware. The data streams from all 16 receivers meet here and each of the $1.28-\mathrm{MHz}$ channels is filtered by dedicated hardware into $128 \times 10 \mathrm{kHz}$ fine channels (Section 2.5). A correlator implemented in software using general-purpose graphical processing units (GPGPUs) processes, averages in time and frequency space, and outputs visibilities in $768 \times$ $40 \mathrm{kHz}$ channels with 0.5 -s resolution (Section 2.5). This 3.2-Gbps stream of correlation products is then processed by the Real-Time System (RTS; Mitchell et al. 2012) to produce real-time calibrated images every $8 \mathrm{~s}$ (Section 2.6). We have enabled the ability for visibility data to also be saved, to allow for the possibility of post-observation processing (Sections 2.6 and 2.7).

The MWA runs a monitor and control system to schedule observations and monitor system health and parameters of use for data processing (Section 2.8).

The RTS output and the $u v$ data are transmitted on a dedicated 10-Gbps optical fibre connection to the Pawsey High Performance Computing Centre for SKA science in Perth, where $15 \mathrm{~PB}$ of data storage capacity has been reserved over a 5-year period to accommodate the MWA data archive (Section 2.7).

Supporting the MWA instrument is the underlying infrastructure, including the reticulation of fibre and power around the array and the connection to MRO site-wide services provided by the CSIRO (Section 2.1).

In the following sections, we provide further detail on the MWA sub-systems.

\subsection{MWA Infrastructure at the MRO}

The MWA support infrastructure at the MRO includes all of the installations and equipment required to transport power and data to, from, and around the array. The focus of the MWA infrastructure is a power and data distribution 'hub' located near the core of the array. This section describes the distribution hub and then, in turn, its connections to central MRO site services and its connections to MWA equipment in the field. All of the infrastructure and equipment described in this paper conforms to the specification 'RFI Standards for Equipment to be Deployed on the MRO. ${ }^{7}$

The hub, located approximately $200 \mathrm{~m}$ south of the MWA core, is the central distribution point for the power and data

\footnotetext{
${ }^{7}$ ASKAP-MRO-0001-Version 1.1, dated 2010 October 15.
}

services into, from, and around the array (Figure 2); it consists of power and data distribution apparatus mounted on separate but adjacent concrete plinths.

A compact substation consisting of a transformer and a low voltage (LV) switchboard housed in an environmental enclosure (kiosk) is mounted on one concrete plinth. All cable entries are from underneath through apertures in the precast concrete plinth. The transformer is a three-phase ONAN type $(\mathrm{O}=$ mineral oil or synthetic insulation fluid with a fire point $300^{\circ} ; \mathrm{N}=$ natural convection flow through cooling equipment and in windings; $\mathrm{A}=$ air is the external cooling medium; $\mathrm{N}=$ natural convection of the external cooling medium) with a ratio of $6.6 \mathrm{kV} / 415 \mathrm{~V}$, a Dyn11 winding arrangement and an impedance of $4 \%$. The incoming cable enters the termination enclosure via an all-metal cable gland which is bonded to the cable sheath. The HV termination enclosure is sealed with conductive RFI gaskets. The LV switchboard comprises the main incoming terminal, including a three-pole manual changeover switch (mains/off/generator) and the outgoing mains feeders. The incoming and outgoing feeders are protected by fused switches. No electronic equipment is employed within the compact substation.

A high-density fibre optic patch panel housed in an environmental enclosure is mounted on a separate but adjacent plinth. All fibre entries are from underneath through apertures in the precast concrete plinth.

The MWA distribution hub is connected to the wider MRO power and data networks by a single incoming services trench. The incoming high-voltage cable is a $35-\mathrm{mm}$ three-core armoured cable with an aluminium conductor and an operating voltage range of $6.35 / 11 \mathrm{kV}$, designed and constructed to comply with AS/NZS1429.1-2006. The incoming data cables are Prysmian Fusion link dry ribbon cables consisting of 18 ribbons of 12 fibres (216 single-mode fibres) operating at $1310 \mathrm{~nm}$. There are two incoming cables providing a total of 432 incoming fibres. An extruded nylon sheath provides environmental protection.

Distribution from the MWA hub is by cables directly buried in service trenches that terminate at service pillars designed to provide direct plug-in points for MWA receivers (and other hardware that can make use of the same service formats). The LV cabling has a copper conductor of various diameters based on the load and length of individual cable runs packaged in flexible cross-linked polyethylene insulation and with an extruded nylon protective barrier. The optical fibre cables consist of 12 cores of standard single-mode fibre in a cable designed for direct burial and with an extruded nylon barrier. The capacity of the fibre and power deployed is designed to accommodate possible expansion of the core of the array at some future point in time.

The service pillars are equipped for single-phase distribution and include a single-phase distribution board used to supply either one or two receivers. The distribution board also provides a local isolation point for the receivers. The service pillars are mounted on concrete plinths with bottom cable entry facilitated by apertures in the precast concrete. 


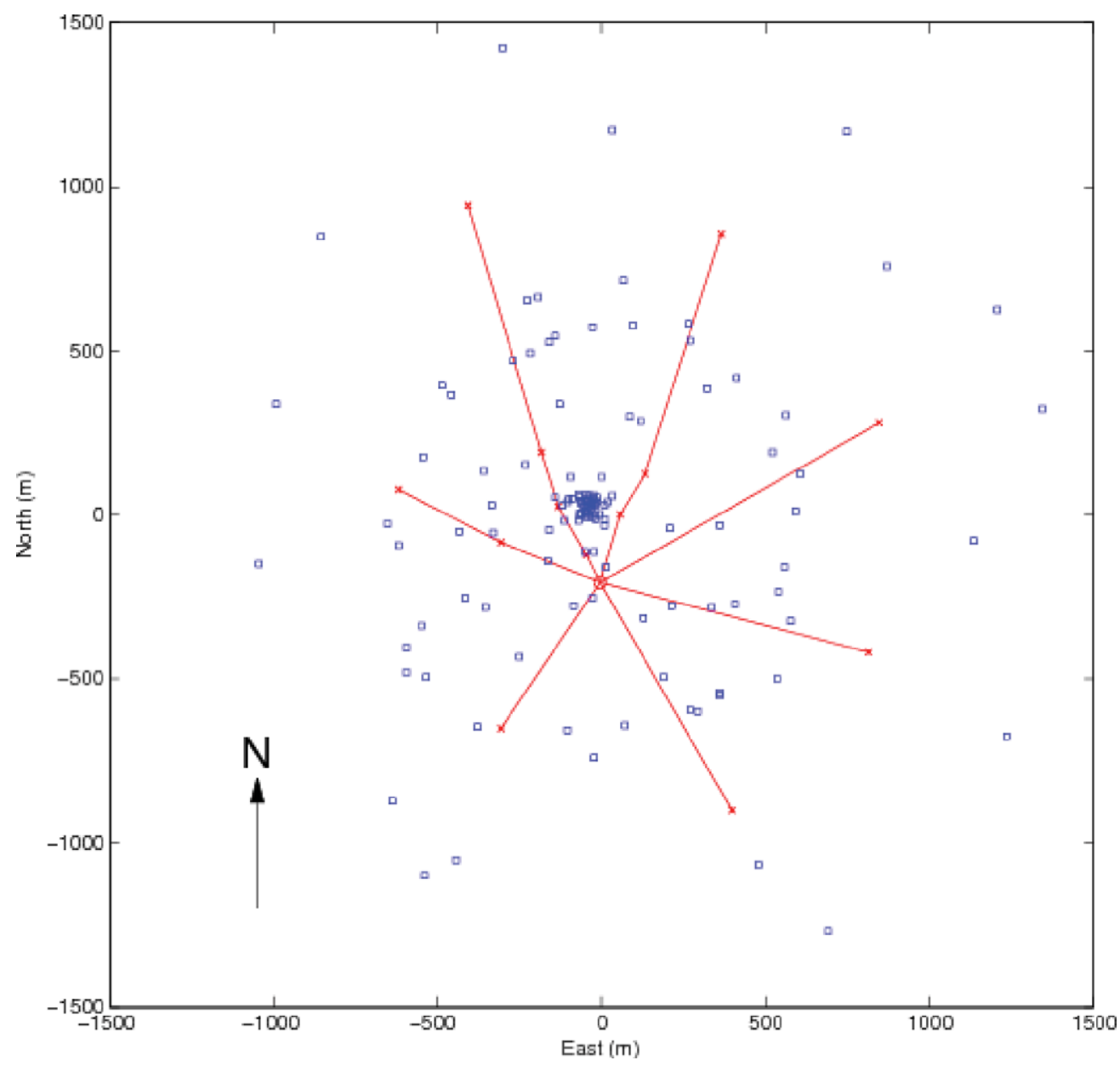

Figure 2. Plan of the MWA infrastructure, as built at the MRO. The hub referred to in the text is located at $(0,-200)$, with seven radial trench lines shown in red. Receiver locations are marked as red crosses and tile positions are marked as blue squares.

The final connection to receiver is by means of a standard three-pin plug and socket.

The CPF houses the MWA post-receiver signal processing equipment within 16 Schroff LHX20 water cooled 19inch cabinets $(42 \mathrm{U}$ height $\times 800 \mathrm{~mm}$ width $\times 1200 \mathrm{~mm}$ depth).

\subsection{Array Configuration}

The initially envisaged MWA design consisted of 512 tiles distributed with a $r^{-2}$ density profile for radii of 50-750 m, with a flat distribution of tiles inside $50 \mathrm{~m}$ and 16 outlying tiles at $\sim 1500$-m radius (Lonsdale et al. 2009). This profile provided smooth $u v$ coverage with a strong concentration of short baselines at the scales relevant for EoR power spectrum measurements (Beardsley et al. 2012), the excellent snapshot imaging needed for transients and holographic calibration (B. Hazelton et al., in preparation).

In re-scoping the array to 128 tiles, we maximised science capacity by keeping the key figures of the original layout: ex- cellent snapshot $u v$ coverage, a concentrated core of tiles for Epoch of Reionisation (EoR) power spectrum measurements, and longer baselines for solar observations. This has led us to place $25 \%$ of the tiles within a dense 100 -m diameter core, with a very smooth tile distribution out to $1.5-\mathrm{km}$ diameter and 16 tiles in an outer region of 3-km diameter. The resulting tile distribution is shown in Figure 3 and the snapshot singlefrequency $u v$ coverage is shown in Figure 4 . The coordinates of the centre of the array are: latitude $-26^{\circ} 42^{\prime} 11^{\prime \prime} .94986$; longitude $116^{\circ} 40^{\prime} 14^{\prime \prime} .93485$; elevation $377.827 \mathrm{~m}$.

Even in the remote Australian desert, there are areas of rock outcroppings, emergency runways, flood zones, and heritage areas where tiles cannot be placed. Beardsley et al. (2012) detail a new tile placement algorithm that produces extremely smooth snapshot $u v$ distributions in the presence of ground exclusion zones. Noting that over- and underdensities in the $u v$ plane act like two-dimensional wave packets to produce PSF (array beam) sidelobes, the new method uses a Bessel decomposition of the $u v$ plane to create very smooth baseline distributions. 
(a)

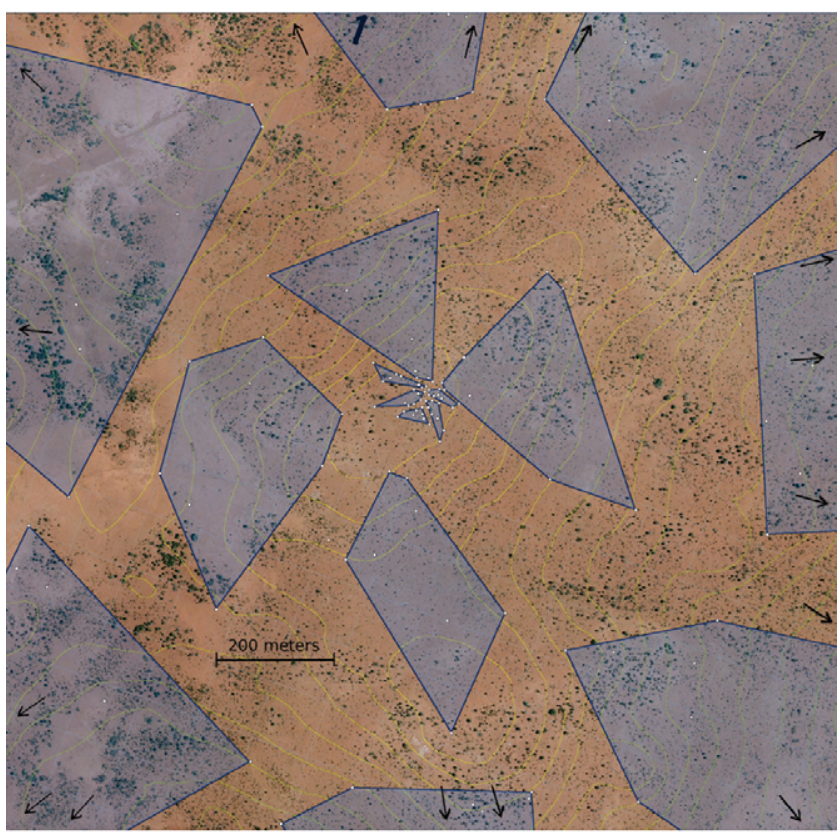

(b)

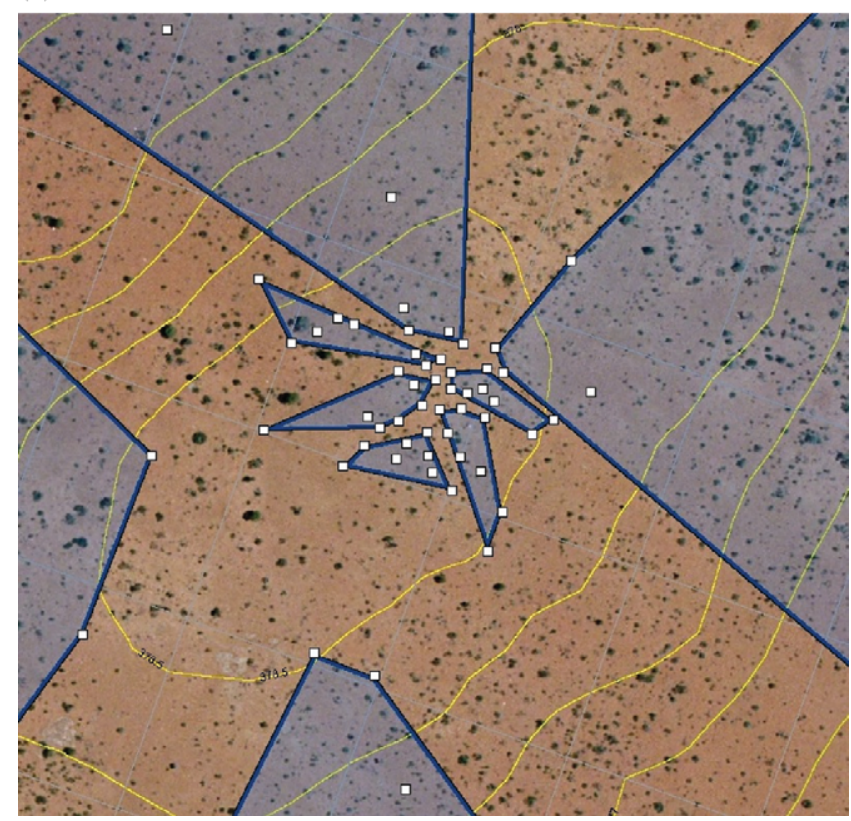

Figure 3. Top: an aerial map of the central 112 antennas of the MWA layout, indicated by white $5 \times 5 \mathrm{~m}$ squares. The layout features a dense core of antennas within $50 \mathrm{~m}$ of the array centre, with a very smooth distribution of antennas out to a $750-\mathrm{m}$ radius. The arrows indicate the direction to the outer 16 antennas for high angular resolution imaging which are in a rough ring of $\sim 1.5-\mathrm{km}$ radius. The purple regions indicate the electrical footprints of each of the 16 receivers, each servicing eight antennas. Bottom: a zoom into the central $\sim 1 \mathrm{~km}$ of the array configuration.

\subsection{Tiles and Analog Beamformers}

The MWA antenna system is composed of the active and passive components described below. These components, when connected together, form a 'tile' and analog beam- (a)
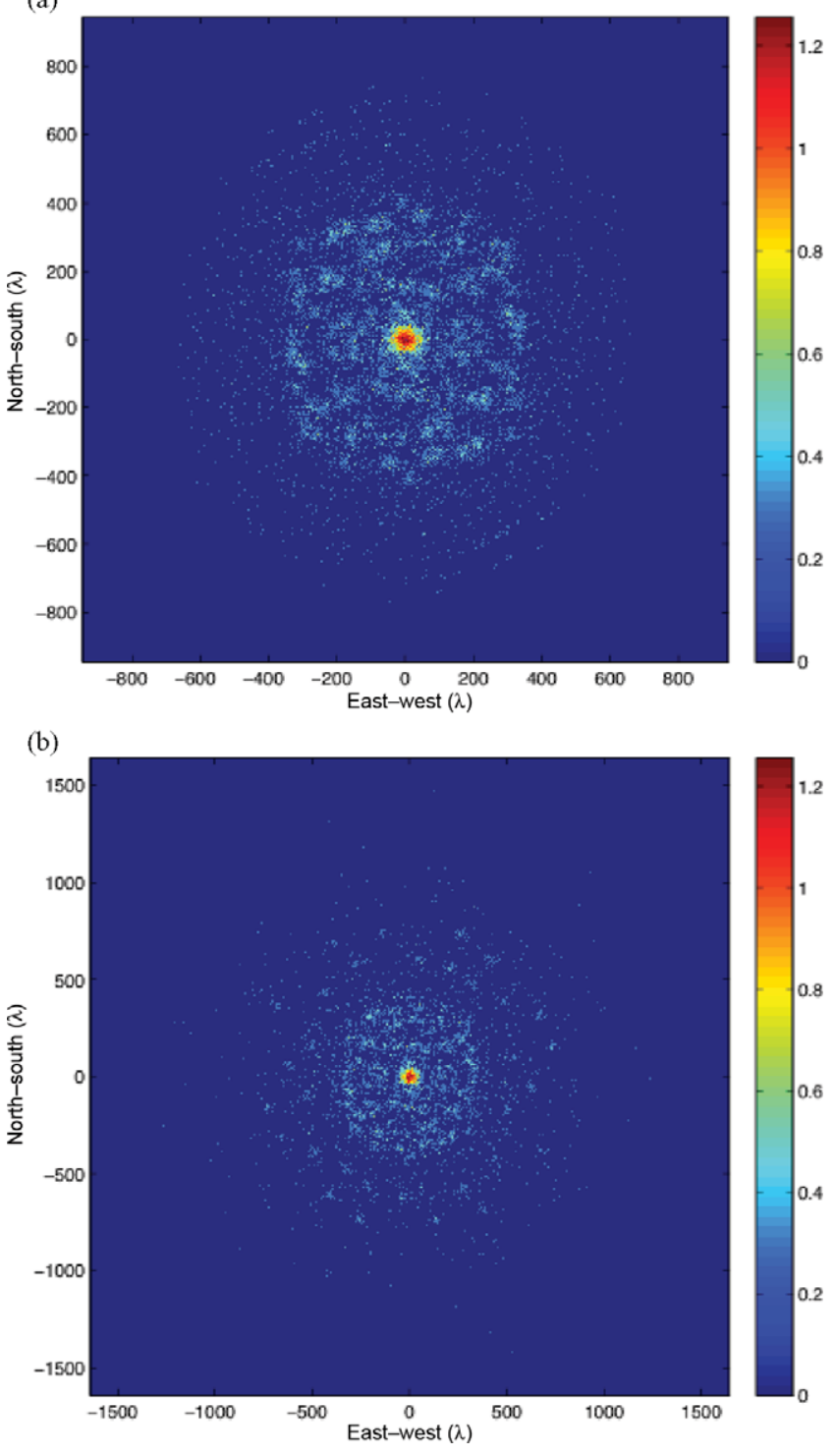

Figure 4. Top: the snapshot of the single-frequency $u v$ coverage for the central 112 antennas. Bottom: the snapshot of the single-frequency $u v$ coverage for the full array.

former (Figures 5 and 6). The MWA has deployed 128 antenna tiles and beamformers, in a configuration described in Section 2.2.

A $5 \times 5 \mathrm{~m}^{2}$ mesh reflecting screen (effectively a plane mirror over the MWA operating frequency range), consisting of a galvanised steel wire mesh with $50 \mathrm{~mm} \times 50 \mathrm{~mm}$ wire spacing and 3.15-mm wire thickness, forms the ground screen for each tile. These ground screens rest directly on the ground, each positioned to align within $2^{\circ}$ of north-south/east-west, within $1^{\circ} .5$ of perpendicular to the zenith, and with less than $5-\mathrm{cm}$ deviations from planarity across each ground screen. The ground screen is composed of three rectangular sheets spot welded together sufficiently well to provide a continuous electrical path and is electrically connected to the metal chassis of the analog beamformer which, through a dedicated 


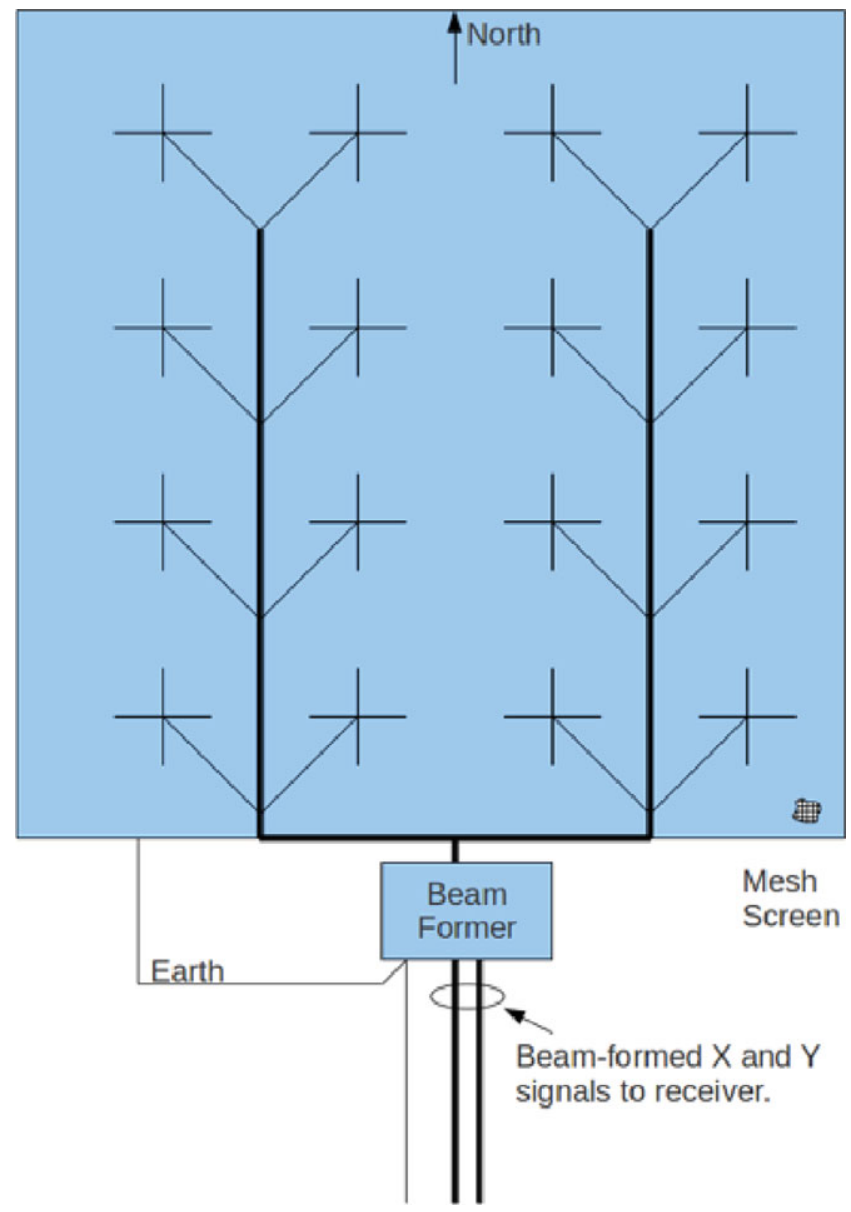

Figure 5. A schematic antenna tile layout. drain wire, is connected to the metal chassis of the receiver, ensuring a good discharge path for static electricity.

Sixteen dual polarisation active antenna elements form a $4 \times 4$ regular grid on the ground screen. Each antenna element consists of two sets of orthogonally mounted aluminium broadband dipole 'bat-wings', each pair directly feeding a custom designed low-noise amplifier (LNA) circuit. The LNA circuit provides approximately $19 \mathrm{~dB}$ of gain at $150 \mathrm{MHz}$ when terminated at $50 \mathrm{ohms}$, and includes a feed conversion from balanced to single ended. The LNA circuit is powered from a 5-VDC bias supplied by the ana$\log$ beamformer. Antenna elements are mounted on the mesh with one dipole aligned north-south and the other east-west. While these active dipoles are broadband by design, the antialiasing filter immediately prior to the digitisation stage in the receiver limits the telescope operating range to approximately 80-300 MHz. The centre-to-centre element spacing on the mesh is $1100 \mathrm{~mm}$ corresponding to half wavelength separation at $136 \mathrm{MHz}$.

The signals from the $16 \times 2$ dipole antennas are wired to the analog beamformer using a 50-ohm cable specified to LMR-100. All these element cables are made to the same length of $7 \mathrm{~m}$ to ensure that all antenna signals reach the analog beamformer front panel with identical delays. As mentioned above, these cables also carry the 5-VDC bias from the beamformer to power the LNA circuits. Each of the 32 channels in the analog beamformer has an independently controlled 32-step delay using five binary-weighted delay steps, each of which can be switched in or out. When any delay step is switched out, a gain-matching circuit stub is switched in to maintain relatively constant gain regardless of delay setting (tile gains are calibrated post-correlation). The delay steps are multiples of 435 picoseconds, allowing

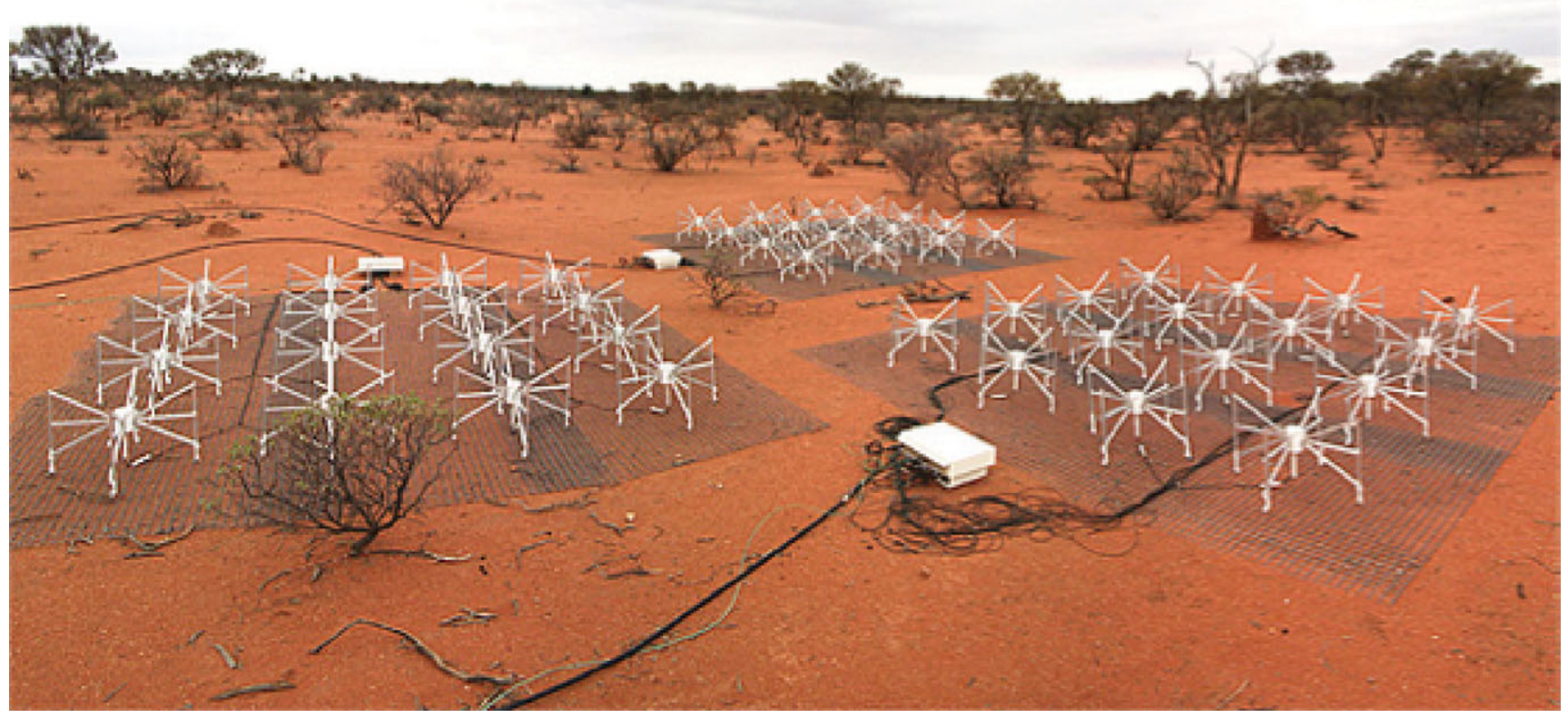

Figure 6. MWA tiles and analog beamformers deployed in the field. 
(a)



(b)

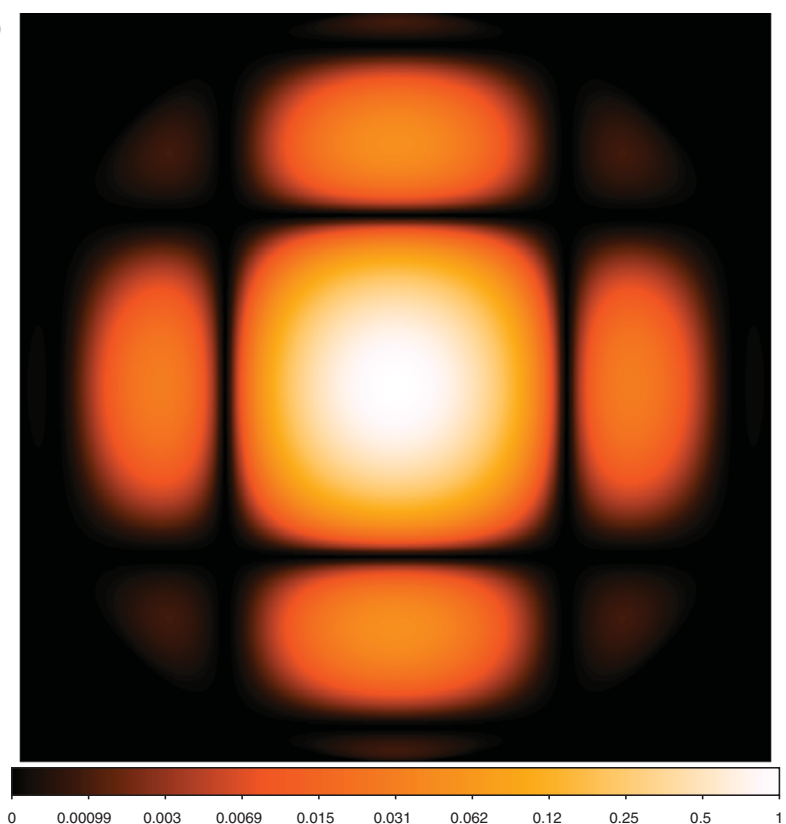

(c)

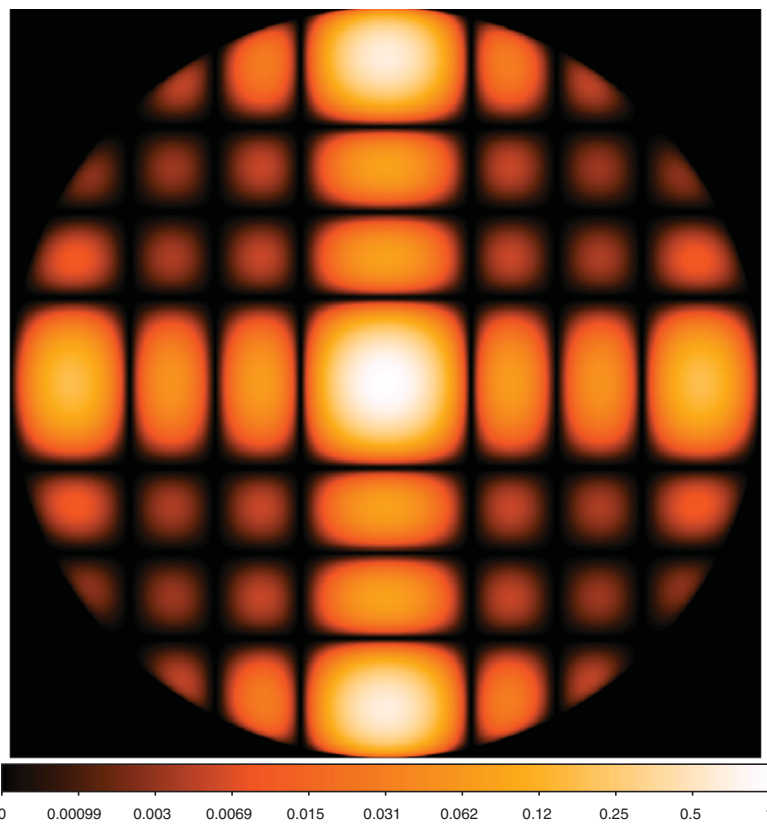

Figure 7. Simulated beam response for a single MWA tile. From left to right, the simulated beams are at observing frequencies of 80 , 150 , and $300 \mathrm{MHz}$, spanning the full frequency range of the MWA. From top left, moving clockwise, the simulated beams note that element cables are not shown to length scale.

a range of discrete relative delays between elements, from zero to $13.5 \mathrm{~ns}$. The outputs from all 16 delay stages for each polarisation are summed to create X- and Y-polarised tile beams on the sky. The analog beamformer, and hence the instrument, can support steering the $\mathrm{X}$ and $\mathrm{Y}$ beams for all 128 tiles in different directions, for a total of 256 beam directions. It should be noted that the beam shape changes with pointing direction (as well as frequency) and pointing directions below $30^{\circ}$ elevation are not available. Figure 7 shows simulated beam responses for a single MWA tile pointed at zenith, spanning the frequency range.
The summed $\mathrm{X}$ and $\mathrm{Y}$ outputs are amplified and impedance converted to match the 75-ohm dual coaxial cable that connects the analog beamformer to the receiver. This cable also carries encoded control signals to set the delays on the analog beamformer, as well as the 48-VDC power for the beamformer. Shorter cables conform to RG-6 specification, while longer cables meet LMR-400-75 specification. Furthermore, on longer cable runs, a 'whitening' filter is inserted to overcome some of the frequency-dependent loss that would otherwise overemphasise the low-frequency signals. 


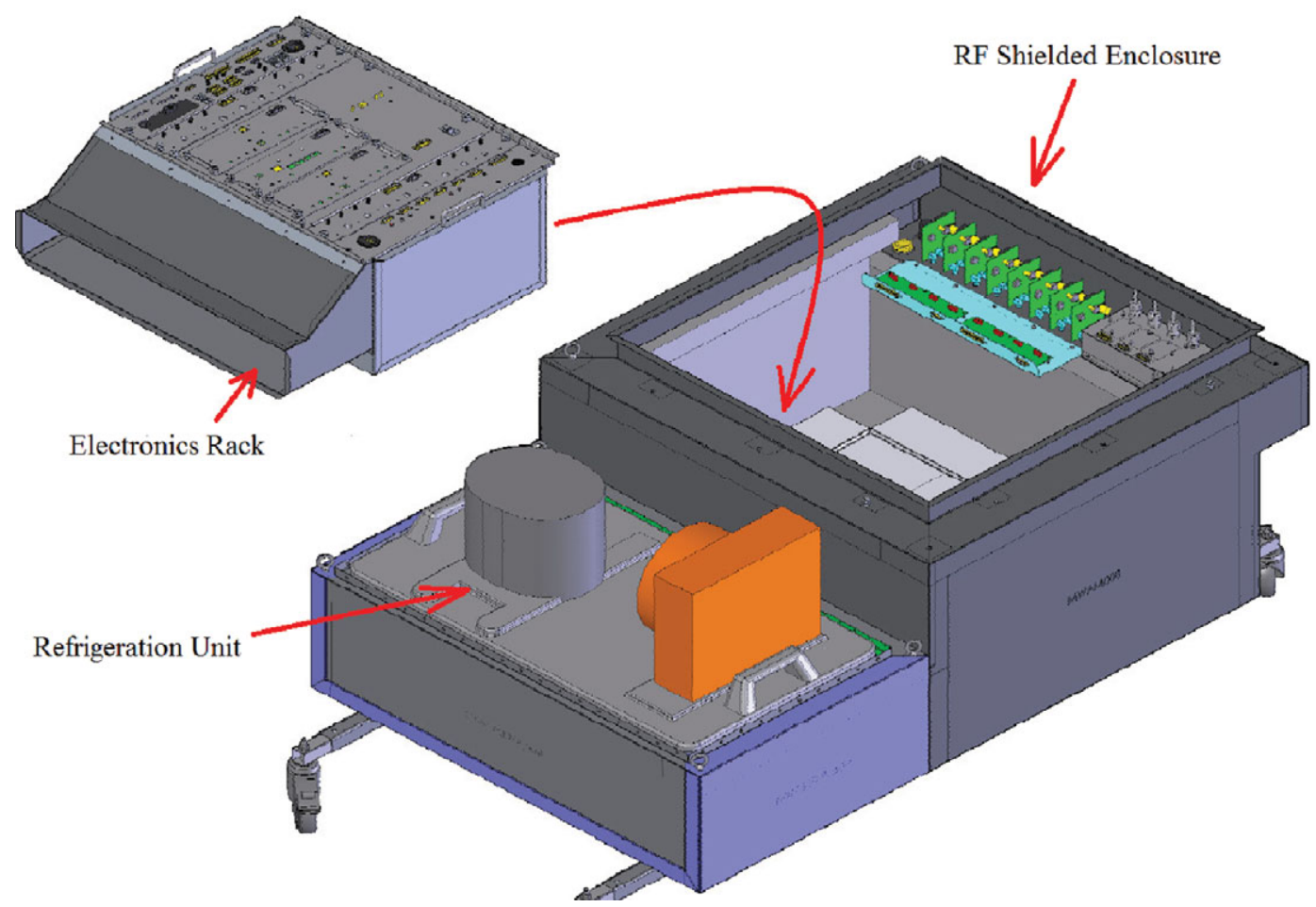

Figure 8. Exploded view of an MWA receiver package.

\subsection{Receivers, Digitisation, and First Stage Channelisation}

An MWA receiver node unit (Figure 8) is responsible for taking the analog radio frequency (RF) signals from eight tiles, performing digitisation and coarse frequency channel selection of these signals, and then transmitting the resulting digital streams via fibre optic cable to the CPF.

There are a total of 16 receiver nodes in the complete 128tile MWA system. These receivers are physically distributed around the array to minimise the length of the coaxial cables that carry the analog RF from the beamformers to the receivers. RF signals arriving at the receiver are passed to an analog signal conditioning (ASC) board where signal-level adjustment, impedance matching, and $80-300 \mathrm{MHz}$ bandpass filtering take place. The conditioned analog signals are then passed to an analog-to-digital and filter bank (ADFB) board. The signals are sampled at 8 bits per sample and 655.36 Msamples s ${ }^{-1}$ by ATMEL AT84AD001B ADC chips and fed to Xilinx Virtex 4 Field Programmable Gate Array (FPGA) chips.

The FPGAs implement a 256-channel coarse polyphase filter bank which gives $256 \times 1.28 \mathrm{MHz}$ wide channels over the $327.68-\mathrm{MHz}$ sampled bandwidth. Of these 256 coarse channels, channel numbers below about 55 and above about 235 are highly attenuated by the ASC filtering.

The channelised data are transferred by a custom-designed high-speed backplane to the Virtex 5 FPGA-based Aggregator Formatter (AGFO) board. A user-defined subset of 24 of these channels (not necessarily contiguous) is formatted and transmitted on three fibre optic cables. Each of the three fibres contains data for eight of the selected coarse channels for each of two polarisations for the eight tiles connected to that receiver. The three fibres together yield $30.72 \mathrm{MHz}$ of RF bandwidth that is transmitted to the CPF. The data are transmitted in the form of $5+5$ bit complex samples. Together, the 16 receiver nodes in the 128-tile system pass in excess of $80 \mathrm{~Gb} \mathrm{~s}^{-1}$ of data to the downstream signal processing systems.

Additional fibres provide Ethernet communications for monitor and control functions and distribute a centralised clock signal for the samplers, FPGA logic, and timing signals for array synchronisation. A single-board computer controls the receiver node functions and services monitor and control (M\&C) needs. Control functions include analog beamformer commands, setting and monitoring of ASC units, configuring and monitoring of high-speed digital boards, thermal control, and managing power start-up and shut-off in response to various conditions.

The receiver electronics are fitted into a rack which is housed in a weather-tight, RF-shielded enclosure with an integrated refrigeration unit. This allows the receiver to meet the stringent radio-quiet requirements of the MRO and to provide environmental conditioning and protection to enable the unit to survive in an environment where the ambient air temperature can range from $0^{\circ}$ to $50^{\circ} \mathrm{C}$.

\subsection{Correlation}

The real-time cross-correlation task for interferometric arrays is a large computational challenge, historically 
addressed by application specific integrated circuits (ASICs) and FPGAs. These technologies are well matched to the limited-precision fixed-point arithmetic required, but their application typically involves trade-offs between cost of development, power consumption, and performance. ASICs are costly to develop and produce; FPGAs are cheaper to develop than ASICs, and both are much more power efficient than general-purpose processors. However, as discussed by Nieuwpoort \& Romein (2009), there has been considerable recent effort expended applying many-core processors to this problem. The MWA has chosen to leverage two technologies to perform the correlation task, a polyphase filtering task (PFB) performed by an FPGA-based solution, and a cross-multiply and accumulate task (XMAC) utilising GPGPU technologies developed by Clark, LaPlante, \& Greenhill (2012).

The purpose of a spectral line correlator is to measure the level of signal correlation between all antenna pairs at different frequencies across the observing band. The result is commonly called the cross power spectrum and for any two antennas $V_{1}$ and $V_{2}$ can be formed in two ways. First, the cross-correlation as a function of lag, $\tau$, can be formed:

$$
\left(V_{1} \star V_{2}\right)(\tau)=\int_{-\infty}^{\infty} V_{1}(t) V_{2}(t-\tau) \mathrm{d} t
$$

The cross power spectrum, $S(v)$, is then obtained by application of the Fourier transform to reveal

$$
S_{12}(\nu)=\int_{-\infty}^{\infty}\left(V_{1} \star V_{2}\right)(\tau) \mathrm{e}^{-\mathrm{i} 2 \pi \nu \tau} \mathrm{d} \tau .
$$

When the tasks required to form the cross power spectrum are performed in this order (lag cross-correlation, followed by Fourier transform), the combined operation is considered an XF correlator. However, the cross-correlation analog of the convolution theorem allows Equation (2) to be written as the product of the Fourier transform of the voltage time series from each antenna:

$$
S_{12}(v)=\int_{-\infty}^{\infty} V_{1}(t) \mathrm{e}^{-\mathrm{i} 2 \pi v t} \mathrm{~d} t \times \int_{-\infty}^{\infty} V_{2}(t) \mathrm{e}^{2 \pi v t} \mathrm{~d} t .
$$

Implemented as Equation (3), the operation is described as an FX correlator. The FX correlator has a large computational advantage. In an XF correlator, the cross-correlation for all baselines requires $\mathrm{O}\left(\mathrm{N}^{2}\right)$ operations for every lag, and there is a one-to-one correspondence between lags and output channels, $\mathrm{F}$, resulting in $\mathrm{O}\left(\mathrm{FN}^{2}\right)$ operations to generate the full set of lags. The Fourier transform requires a further $\mathrm{O}\left(\mathrm{F} \log _{2} \mathrm{~F}\right)$ operations, but this can be performed after averaging the lag spectrum and is therefore inconsequential. For the FX correlator, we require $\mathrm{O}\left(\mathrm{N} \log _{2} \mathrm{~F}\right)$ operations per sample for the Fourier transform of each data stream, but only $\mathrm{O}\left(\mathrm{N}^{2}\right)$ operations per sample for the cross-multiply (although we have F channels, the sample rate is now lower by the same factor). As discussed by Clark et al. (2012), there is a huge computational advantage in implementing an FX correlator of at least a factor of F. However, XF correlators have been historically favoured by the astronomy community, at least in real-time applications, as there are disadvantages of the FX implementation. The predominant disadvantage is data growth: the precision of the output from the Fourier transform is generally larger than the input, resulting in a data rate increase, and there is also the complexity of implementing the Fourier transform.

The MWA implements a hybrid, distributed FX correlator solution that efficiently deals with the disadvantages of an FX correlator (Figure 9). The F stage is performed in two stages: first as a coarse, complex filter in the receiver, as described in the previous section, and then to finer spectral resolution by four dedicated FPGA-based polyphase filter bank (PFB) boards co-located with the cross-multiply system in the CPF. The fine channelisation system captures 48 fibres in total from 16 receiver nodes; each fibre carries $24 \times 1.28 \mathrm{MHz}$ spectral channels from both polarisations of eight antenna tiles digitised to 5-bit precision. An individual PFB board ingests the full bandwidth from four receivers (32 tiles) and performs a further factor of 128 in channelisation, resulting in $3072 \times 10 \mathrm{kHz}$ channels. The output of each PFB is therefore 3072 Nyquist sampled, 10-kHz channels from two polarisations of 32 tiles, presented on serial data lines via an interface module known as the rear transition module (RTM). The last stage of the PFB is a bit-selection stage that selects only the most significant four bits to represent the samples. This effectively counters the major disadvantage of the FX correlator, data growth. In the MWA case, as 5-bit precision is input and 4-bit precision is output, the $\mathrm{F}$ stage performs a slight reduction in data rate.

The MWA cross-multiplication and accumulate (XMAC) is frequency multiplexed and performed independently on 24 IBM iDataPlex dual Xeon servers, each housing $2 \times$ NVIDIA Tesla M2070 Graphics Processing Units. Each machine is allocated 128 contiguous $10-\mathrm{kHz}$ channels from all the antennas. A Chelsio 10-GbE interface card and a 64port IBM BNT 8264 switch are used to provide the packet switching required to aggregate the data from each PFB output. To permit packet switching, the output of the PFB must be converted from the Xilinx propriety serial protocol (RocketIO) into $10 \mathrm{GbE}$. The media conversion is performed by a set of 16 dedicated dual Xeon servers, each housing a Xilinx FPGA-based capture board (supplied by EDT Incorporated, Beaverton, Oregon, USA) which accepts a portion of the serial data from the PFB and presents it to system memory where a bespoke software application routes each packet to a target machine. A connection-based protocol (TCP) is used due to the complex nature of the packet switching and the synchronisation issues arising from capturing many parallel streams in a general-purpose computing environment.

Although the purpose of the operations described above is to facilitate re-packetisation from RocketIO to $10 \mathrm{GbE}$, the 16 servers also provide a capability to capture the voltage samples directly to disk. The voltage capture system (VCS) will record a copy of the voltages using a continuous ring buffer via a SAS-2 controller in each server with enough 


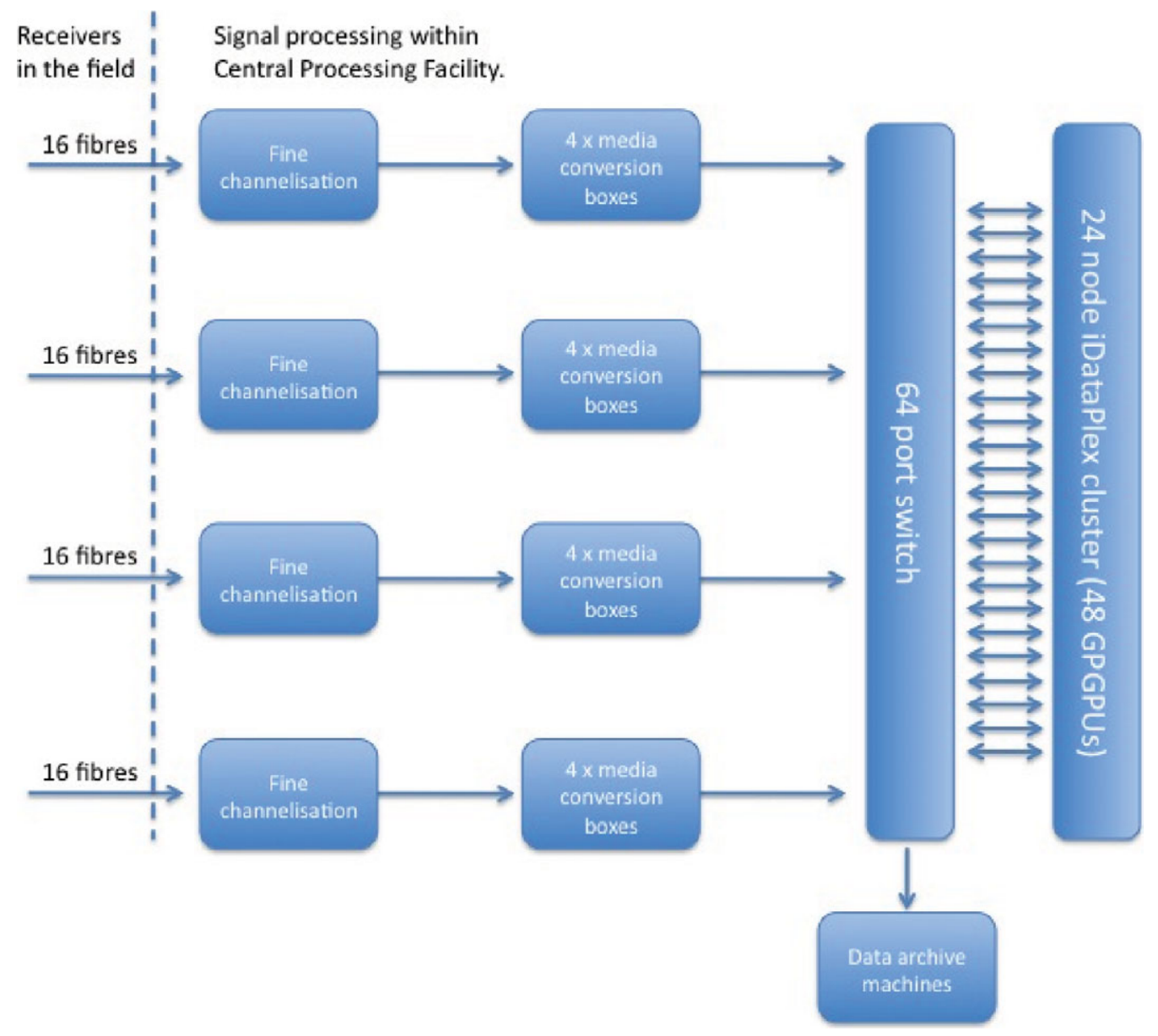

Figure 9. Correlation sub-system overview.

$10000 \mathrm{rpm}$ hard drives to store an hour of data at minimum. Upon receiving a trigger, the ring buffers will form new rings using unused memory on the drives, thus preserving the data from the previous buffers. The VCS servers contain enough memory to store at least 4 min of data which can be searched through for transients with inherent timescales shorter than the integration time of the telescope using incoherent dispersion and to prompt the trigger on the ring buffer when a detection occurs. Alternatively, an external trigger can be used to save the data from the ring buffer. Room has been intentionally reserved within this system to incorporate realtime GPU processing at a later date. The recorded voltages can be correlated using existing software correlation solutions, such as DiFX (Deller et al. 2011, 2007).

In order to obtain data from all the tiles for its frequency allocation, each of the 24 XMAC servers has to accept packets from all of the media converters and obtain them in the correct order within a narrow time interval. This task is enabled by extensive use of parallel programming methodologies. Despite the large aggregate bandwidth, the bandwidth per XMAC machine is only $2.8 \mathrm{Gbps}$ and is well within the PCIe2.0 bandwidth of 64 Gbps available to the 16-lane Telsla M2070. The GPU-enabled XMAC operation is described in detail by Clark et al. (2012) and the level of computation required to process $1.28 \mathrm{MHz}$ of bandwidth for 128 tiles is 335 GFLOP, which is also well within the capability of the XMAC kernel we employ. The XMAC is fully performed within the GPU environment, with an output resolution of $0.5 \mathrm{~s}$ and a channel resolution of $10 \mathrm{kHz}$ (which will be combined to $40 \mathrm{kHz}$ to facilitate storage). The format is half the correlation matrix for each channel with a lightweight FITS (Wells, Greisen, \& Harten 1981) header and the output data rate from the XMAC is 3.2 Gbps.

\subsection{Real-Time Imaging and Calibration}

The ever-growing data rates generated by next-generation radio arrays, in the MWA case driven by the wide fields of view and correlation-rich architecture, are forcing astronomers to integrate visibilities over longer time intervals than are otherwise desirable. One approach to dealing with these problems is to make snapshot images and to extend data reduction into the image domain, where various types of directiondependent corrections can be readily made.

The MWA sub-systems outlined in previous sections contain novel elements, such as wide-field fixed dipole antennas and GPU-based software correlation, but describe signal chain operations that are largely traditional in nature. 


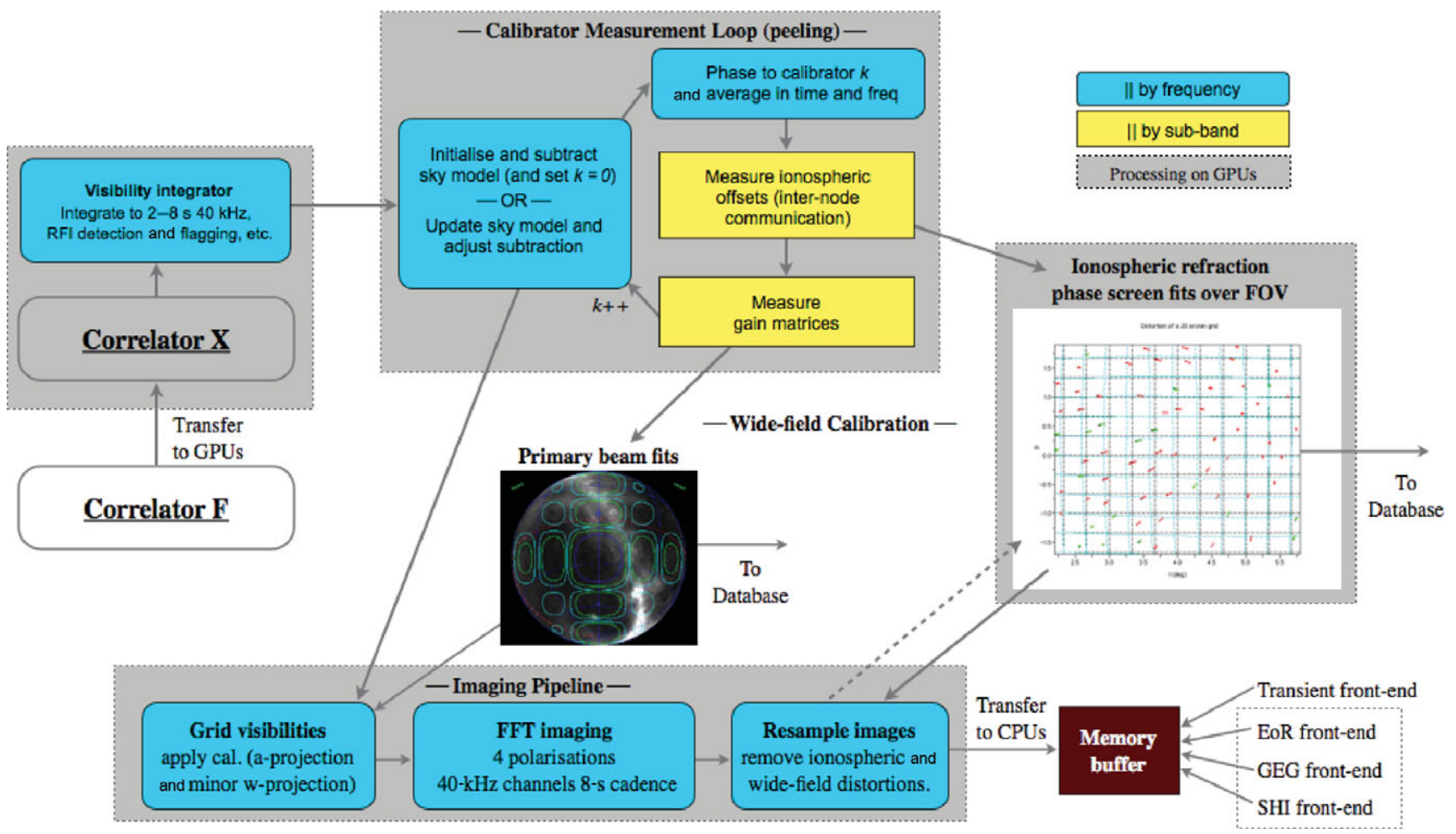

Figure 10. Overview of the MWA RTS, as described in the text.

Real-time imaging and calibration systems at the correlator output are emerging as a critical element of next-generation wide FoV arrays, for example as demonstrated for LOFAR M. van Harlem et al. (in preparation) and as planned for ASKAP (Johnston et al. 2008, 2007). As such, we include below a relatively extensive discussion of the MWA approach to this sub-system (RTS) and function. Figure 10 presents a schematic overview of the following description.

Synthesis imaging is centred around inverting the threedimensional van Cittert-Zernike theorem, which describes the transformation between the desired sky brightness distribution and the measured visibilities (Thompson, Moran, $\&$ Swenson 2001). Solutions to this problem for arrays like the MWA are challenging, due mainly to four issues that worsen with increasing FoV size: how best to deal with the three-dimensional problem in a way that is computationally feasible and conducive to high-dynamic-range deconvolution; how best to deal with an ionosphere that causes timeand direction-dependent distortions; how best to deal with antennas that have primary beam and polarised feed configuration variations in time, frequency, and direction, and that differ from one antenna to the next; and how best to deal with the large data rates and data volumes. For an approximately coplanar array such as the MWA, an imaging approach based on snapshots can meet all of these challenges: one can think of snapshot visibilities as a slice through the three-dimensional visibility set, for which the ionosphere and the nominal feed configuration matrices towards each source are constant. They are also consecutive in time and so are well suited to pipeline processing. We will consider each of the issues separately.

For a sufficiently short snapshot observation, and at the expense of a non-uniform coordinate distortion in the sky brightness distribution, the transformation between sky brightness and the visibilities of a coplanar array reduces to two dimensions (Bracewell 1984; Cornwell \& Perley 1992; Perley 1999; Cornwell, Golap, \& Bhatnagar 2005; Ord et al. 2010). The non-uniform coordinate distortion comes from imaging on a plane that is parallel to the array plane, rather than the tangent plane at the field centre, and thus it will change as the field centre is tracked. Once these coordinate distortions have been removed, which requires an image resampling process, time integration can continue in the image domain after appropriate pixel-wise weighting to maximise the signal-to-noise ratio $(\mathrm{S} / \mathrm{N})$. This is known as warpedsnapshot imaging, and in the RTS the procedure is combined with ionospheric corrections and conversion to right ascension and declination coordinates (in the HEALPIX pixelisation scheme; Gorski et al. 2005).

Averaging in time is computationally expensive when it is done in the image domain, and in many traditional situations the pixel-based operations will dominate the processing, often by orders of magnitude. For arrays such as the MWA, however, as the number of visibilities grows relative to the number of pixels, it can become competitive or even cheap compared with alternatives such as W-projection (Cornwell, Golap, \& Bhatnagar 2005). On the other hand, removing the non-uniform warp from the snapshot images will lead to a 
shift-variant point spread function (PSF), which can significantly affect deconvolution, as described by Perley (1999). However, the variable primary beams and the variable atmosphere described below, which are realities for most lowfrequency dipole arrays, can lead to variable PSFs, both in position and time, and joint-deconvolution approaches will likely be required, regardless of the imaging strategy. In their recent paper, Cornwell, Voronkov, \& Humphreys (2012) compare the performance and quality of these approaches and describe a hybrid method that has advantages for arrays such as the SKA. Such an approach is a natural extension to the RTS; however, we note that the maximum snapshot averaging time will be governed by the ionosphere.

One of the most compelling reasons to make snapshot images is the ionosphere. During good ionospheric conditions, the size scales of the ionosphere that are expected to be relevant for the MWA are large relative to MWA baseline lengths (Lonsdale et al. 2009). The effects of the ionosphere in such a situation reduce to direction-dependent shifts in source positions, and direction-dependent Faraday rotation that is constant across the array. While it is possible to deal with the time-dependent ionospheric distortions in the Fourier domain, the angular variations expected for the MWA will require either very large gridding kernels or many image facets. In a warped-snapshot imaging approach, position distortions can be removed during the warped pixel re-sampling step, while ionospheric Faraday rotation can be taken into account during pixel-by-pixel polarisation conversion. In the RTS, the distortions are modelled by interpolating between position measurements of many compact sources with known positions, at the imaging cadence (i.e. every $8 \mathrm{~s}$ ). It is unclear how well we will be able to measure ionospheric Faraday rotation, but an array of GPS receivers is being tested for this purpose, in a similar manner to the approach of Erickson et al. (2001), and we note that we only need to correct changes in ionospheric effects that occur within the image averaging time interval (i.e. several minutes). Any information that becomes available later (by carefully re-processing the data for polarised sources, from other instruments, etc.) can be incorporated during post-processing.

Primary beams that differ from visibility to visibility (due, for example, to changes in time or differences between antennas) are not just of concern for low-frequency dipole arrays. They are a part of wide FoV imaging where a high dynamic range is required. Making direction-dependent corrections at the sample level is possible if one incorporates primary beam information into the visibility gridding kernels, an approach that is being investigated by a number of groups (Myers et al. 2003; Bhatnagar et al. 2008; Morales \& Matejek 2009; Smirnov 2011; Mitchell et al. 2012). These approaches typically require larger gridding kernels, which can be computationally demanding. This type of gridding has the benefit of providing the pixel-wise weighting required for optimal snapshot integration (Ord et al. 2010). Arrays of dipoles have an added complication, however, in that the Jones matrices that convert between sky and instrument polarisations are both time- and direction-dependent (Mitchell et al. 2012). These transformations are difficult to correct for in Fourier space when large FoVs are involved, and will also lead to large convolution kernels or will require many facets on the sky. The RTS approach is a good fit to this problem, since any direction-dependent coordinate corrections can be made in the image domain. When generating visibility gridding kernels, the RTS uses a unique primary beam model for each snapshot, frequency, polarisation, and antenna. It also has the ability to update the models in real time, using measurements of antenna gain towards all strong, compact sources, which are described later in this section.

Finally, a few points should be made about real-time averaging of gridded data. The RTS will average images over several minutes, storing the resultant integrated images. During each interval, the ionospheric and coordinate variations described above are typically fairly small, and the larger variations that occur over hours can be dealt with in postprocessing. Furthermore, since we grid for a direction that is normal to the plane of the array, the size of the gridding kernels can be kept at a minimum. This is not the case for approaches cited above, in which most of the corrections occur during gridding, and so would need to occur in real time. The task is still extremely computationally demanding, and the RTS has been designed and written to run on high-performance GPUs (Edgar et al. 2010; Ord et al. 2009).

From the discussion above, it should be clear that the real-time calibration system has two main tasks: it needs to measure primary beam patterns, and it needs to measure ionospheric distortions across the FoV. To avoid pixelisation effects during deconvolution, it will also subtract strong compact sources from the visibilities, before gridding (peeling, as described by Noordam 2004). Each GPU will process and make images for a small number of adjacent frequency channels, as described in detail by Mitchell et al. (2008) and Edgar et al. (2010), and the baseline system for real-time processing at the MWA is 32 consecutive $40-\mathrm{kHz}$ channels.

Twice per second, a complete set of visibilities arrive from the correlator, split over 24 iDataPlex compute nodes in subbands of $1.28 \mathrm{MHz}$ (the RTS is implemented on the same machines as the correlator cross-multiplication, sharing the GPU resources between the correlator and RTS). The visibilities for each baseline are averaged in time and frequency over the longest intervals for which decorrelation from delay and fringe rates remains below $1 \%$, which is nominally $40 \mathrm{kHz}$ and either 2, 4, or $8 \mathrm{~s}$, depending on the baseline length. After averaging, the RTS performs a series of standard calibration tasks, such as applying cable corrections, automatic RFI detection (as described by Mitchell et al. 2010) and flagging, and then the visibilities are sent to the GPUs. The data are now ready to be used to solve for the instrumental and atmospheric gains and phases, a non-linear optimisation problem that we approach via peeling, as outlined in the list below. For the MWA, much of the visibility noise comes from the sky itself. Diffuse emission will be correlated on short baselines, and these are down-weighted during calibration. 
Model visibilities are also generated from antenna primary beam models and a sky model, and are subtracted to generate residual visibilities. At minimum, the sky model will include all of the strong compact sources, which will be added back in turn-ranked based on the amount of power they contribute to the visibilities - for the following calibration steps:

1. Rotate visibilities: The visibility phases are rotated to be centred at the estimated calibrator position, and the visibilities for each baseline are averaged across all available time and frequency samples (i.e. averaged to $8 \mathrm{~s}$ and $32 \times 40 \mathrm{kHz}$ ), into a temporary working visibility set.

2. Ionospheric measurements: A position offset for the calibrator is modelled as a baseline- and frequencydependent offset in the imaginary part of each visibility, as in Mitchell et al. (2008), which will be zeromean noise if the source is at the phase centre. A single pan-frequency measurement is made to help isolate ionospheric phases from any uncalibrated instrument phases.

3. Instrumental gain measurements: A Jones matrix for each antenna is determined towards the calibrator, based on the least-squares approach discussed by Hamaker (2000) and Mitchell et al. (2008). At present, a running mean is used to reduce sidelobes from other sources and noise; however, an improved approach that uses a Kalman filter is under investigation. Weaker sources may require pan-frequency measurements, as is done with the ionospheric fits. For the most dominant calibrator, the optimisation is repeated for each frequency channel, and polynomial fits to the resulting Jones matrices are used for bandpass calibration.

4. Source subtraction: If the gain and ionosphere measurements pass a set of goodness-of-fit tests, they are used to peel the source from the full-resolution visibility set, thus updating the initial source subtraction. Otherwise, the initial source subtraction is repeated.

5. If there are more calibrators in the list, loop back to step 1 .

The output of this loop, apart from the peeled visibilities, is a set of ionospheric offset measurements and a set of Jones matrix measurements for each tile, distributed in angle across the FoV and sidelobes. The ionospheric measurements are used to adjust the pixel boundaries used in the warped pixel re-sampling step, and we currently use a moving leastsquares approach to interpolate between the calibrators. For isoplanatic patch sizes of $\sim 4^{\circ}$ and image sizes of $\sim 30^{\circ}$, we will need at least 50 or 60 sources in the field to describe the phase variation of each patch. Optimally, we would like to oversample these variations by a factor of at least a few, and if needed we will make position measurements in the images themselves to increase the number of sources. A LevenbergMarquardt approach is used to fit primary beam models, the form of which will be finalised during commissioning. We expect to make Jones matrix calibration measurements for at least a few dozen sources at the imaging cadence.

For the MWA and other similar arrays, the process known as deconvolution is not really deconvolution, since the PSF changes with time, position, and polarisation. However, with various modifications, many of the standard deconvolution approaches can be used to accurately remove PSF sidelobes, and developments are being made on a number of complementary fronts, see for example Pindor et al. (2011), Bernardi et al. (2011), Williams et al. (2012), I. Sullivan et al. (2012), Mitchell et al. (2012), and G. Bernardi et al. (2012).

Finally, one of the biggest risks involved with real-time calibration and imaging is that the visibility data volume is too large to store. The ability to loop back to the raw visibilities during deconvolution is at the heart of traditional high-dynamic-range synthesis imaging and is lost in such a system. The MWA approaches high-dynamic-range imaging from a non-traditional angle, that of a densely filled aperture that has a high-quality instantaneous PSF with low sidelobes. This is important for both deconvolution and real-time calibration, and also allows us to limit the amount of PSF variation from snapshot to snapshot.

However, it is unclear how well this approach will compare with more traditional self-calibration approaches, and to ensure the best quality images, the MWA project will also store visibility data at the full resolution of the correlator $(0.5 \mathrm{~s}$ and $40 \mathrm{kHz})$. Comparisons of image quality will be made using the RTS running in real time, using the RTS running on stored data in a full self-calibration and deconvolution loop, and using other imaging strategies available in standard software packages. The RTS development and evaluation will make the MWA an important step in the development of low-frequency radio astronomy in the lead-up to the SKA.

\subsection{Offsite Data Transport and the MWA Data Archive}

The output of the RTS (calibrated images) and the visibility output of the correlator, plus miscellaneous control data and calibration information, totals approximately $4.4 \mathrm{Gbps}$ at maximum data rate. These data are streamed to a dedicated 10-Gbps connection implemented on the CSIRO optical fibre network between the MRO and Geraldton, which is seamlessly integrated onto an AARNet network dedicated to carrying data traffic from the MRO to Perth. The termination point of the connection in Perth is iVEC, which hosts the Pawsey HPC centre for SKA Science (aka the Pawsey Centre), a new \$A80-million supercomputing centre. Up to 15 PB of data storage has been allocated at the Pawsey Centre, ramping up over a 5-year period from 2013, to host the MWA data archive (both images and visibility data). At the full data rate, this storage allowance corresponds to approximately $300 \times 24 \mathrm{~h}$ of observation. In practice, some percentage of the MWA observations will be at a significantly lower data rate, thus corresponding to more than 300 days 
of observations. Since the visibility data will be archived, it will be possible to reprocess visibilities in a post-observation mode, using Pawsey Centre or other resources (organised by users) and the RTS or other software packages. This will allow gradual refinement of imaging and calibration techniques and the highest quality data products.

The Pawsey Centre is currently under construction. However, the MWA data archive has been specified and a prototype archive system has been implemented for testing and to support commissioning and early operations. The MWA data archive will utilise the NGAS software (Wicenec \& Knudstrup 2007) and is implemented on a single server machine. The server includes a storage array consisting of $24 \times 2 \mathrm{~TB}$ drives arranged in a RAID5 configuration for redundancy. The total amount of storage is $\sim 48 \mathrm{~TB}$ when parity and striping are taken into account. The storage capacity is expandable up to $\sim 170 \mathrm{~TB}$ if required. There are 24 separate 1 -Gbps links from each of the correlator/RTS nodes that are multiplexed into the storage array at a peak sustained data rate of $\sim 3.2 \mathrm{Gbps}$ for visibility data and $\sim 1.2 \mathrm{Gbps}$ for RTS data, combining to a data rate of $\sim 4.4$ Gbps while observing. This equates to $\sim 24 \mathrm{~h}$ of continuous observing time at a 32-bit real and imaginary precision, 0.5 -s integrations and $40-\mathrm{kHz}$ course channel configuration. Data throughput tests have shown that NGAS is capable of streaming to the RAID5 disk array at a rate of $\sim 6.4$ Gbps. The remaining bandwidth will be dedicated to management links required for operations.

The NGAS archive has been engineered to allow file formats to change over time or new data pipelines to be installed without the need to change code or modify the underlying archive software configuration in any way. A data capture application programmer interface has been developed to be the entry point into the archive that can be embedded into any application to accommodate future data pipelines. Engineers and scientists will have access to a web-based interface that is the portal into the archive. For a given set of observation search results, users will be presented with links to data files and their associated metadata. The archive will offer the functionality to convert raw visibility data into a standard UV-FITS format. Such tools will include the capability to greatly reduce the bandwidth and processing overheads for each user. Users will have the option to access and download the raw visibility data if required.

\subsection{Instrument Monitor and Control}

The large number of tiles and the ubiquity of embedded processing have led us to implement a highly distributed monitor and control (M\&C) system for the MWA. Most interferometer $\mathrm{M} \& \mathrm{C}$ systems are monolithic in design with a tight command and control paradigm for each antenna and hardware system. In contrast, the MWA M\&C system is a much more distributed design that leverages the embedded processing in each hardware system. In essence, each local hardware controller is responsible for the health and safety of that instrument system and all of the detailed hardware state changes (e.g. power-up sequence and order of digital mixer changes to change the receiver frequency). The central portions of the $\mathrm{M} \& \mathrm{C}$ are then primarily concerned with orchestrating all of the hardware for observing and commissioning and providing a central repository for all of the configuration and monitoring information (metadata).

Part of this change is due to the ubiquity of embedded processing - even power outlets now have embedded webservers-so it is reasonable to expect every system that interfaces with $M \& C$ to have the local intelligence to perform low-level operational tasks. The other driving factor is the sheer number of tiles and field hardware systems. As the number of antennas reaches into the hundreds, it becomes more difficult to maintain centralised control. Instead of trying to have a single entity simultaneously control hundreds of sub-systems, we have opted for a model where many individual entities are coordinated centrally. The central coordinator directs the distributed entities at a high level and these entities are responsible for controlling and monitoring the given sub-systems.

At the heart of the MWA M\&C system is a relational database that serves as a central repository of all system information. This database includes current and historical configuration; the planned observing schedule (by GPS second), the detailed state changes of each hardware and software system; monitoring data; and links to associate data files with the schedule, configuration, and all associated commands and state changes.

This central database allows us to accurately reconstruct the state of the array for any observation, including a large volume of debug information. For example, we record the full broadband $80-300 \mathrm{MHz}$ spectrum for each tile every $\sim 8 \mathrm{~s}$. Comparing the spectra of different tiles and crossreferencing with changes in pointing direction can quickly diagnose many errors, even when they only happen sporadically. The database is implemented in Postgres, and all of the other M\&C systems interact directly or indirectly with the database.

The other major M\&C systems are the following:

- The Scheduler loads the desired observing schedule into the database. As noted below, there are many settings which can be either set specifically or delayed until run time (e.g. use all of the antennas in the list of good antennas). The Scheduler is implemented in a scriptable Python library.

- The array configuration web pages allow the entry and modification of the array configuration information. This includes the location, serial numbers, and interconnections of all the major hardware components and includes specific pages for common configuration changes (e.g. moving a beamformer from one tile to another).

- Each hardware and software component has a local control program. It receives commands, implements those commands at the indicated clock tick (synchronised by 
the array clock and PPS), and records all of its state changes in the central database.

- The Observation Controller serves as the conductor, and reads the schedule and the configuration tables of the database and sends the appropriate commands to the field hardware. All commands are sent in advance with the GPS time of when the command should be implemented to allow clock accurate commands despite the $\sim 10$-ms jitter inherent in any messaging system. The amount of advance notice is part of the interface definition for each hardware to Observation Controller relationship. The Observation Controller also resolves any observation-time decisions. For example, this allows the schedule to be written weeks in advance to use only the tiles the instrument team judge as good, and which tiles are actually in that list are resolved at observation time. Even within an observation, as tiles are added and removed from the list, the tiles included in the observation are dynamically adjusted. The Observation Controller is implemented in Java.

- The Facility Controller serves a similar purpose to the Observation Controller, but concentrates on startup and shutdown of the array, software and firmware updates, and restarting faulty equipment. While this could have been implemented as part of the Observation Controller, the staged startup of the array (e.g. making sure the necessary network switches have started prior to booting a receiver, and staging the receiver startup to limit inrush current) and software updates are conceptually quite different than observation commands and we have separated the implementation of these functions. The Facility Controller is implemented in Python.

- The Monitoring Tools present the current and historical state of the array to users. All of the system information is stored in the central database, but these tools present the database information to the user in a useful way. A few examples include: a page that lists all of the data files produced during an observation and a summary of all the receivers and tiles that were online; a zoomable plot of the temperature of each tile versus time ( ambient temperature) or each receiver versus time ( $\sim$ receiver air conditioner performance); a plot of the broadband $80-300 \mathrm{MHz}$ spectra during the course of an observation; results of a commissioning observation that turns on one dipole at a time for each tile to measure the complex gain of each dipole as a function of frequency. The Monitoring Tools are implemented as a diverse and growing set of web tools.

- The Police are a set of tests that determine when a system is performing within specifications. They range from very simple (is a receiver responding to commands) to quite complex (identifying unstable LNAs from the broadband spectra). The results of the Police are logged back to the database and are the basis of alerts presented by the Monitoring Tools (turning a receiver status from green to red) and can be used to dynamically
Table 2. Sensitivity of the MWA at $150 \mathrm{MHz}$

\begin{tabular}{lcc}
\hline \hline Property & $B, \sigma_{\mathrm{s}},\left(\Theta_{B}\right)$ & Value \\
\hline Surface brightness sensitivity & $1 \mathrm{MHz}, 1 \mathrm{~K},\left(1^{\circ}\right)$ & $60 \mathrm{~s}$ \\
Point source sensitivity & $1 \mathrm{MHz}, 10 \mathrm{mJy}$ & $800 \mathrm{~s}$ \\
Point source sensitivity & $31 \mathrm{MHz}, 10 \mathrm{mJy}$ & $26 \mathrm{~s}$ \\
Broadband survey speed & $31 \mathrm{MHz}, 10 \mathrm{mJy}$ & $1.5 \times 10^{5} \mathrm{deg}^{2} \mathrm{~h}^{-1}$ \\
Narrowband survey speed & $0.04 \mathrm{MHz}, 10 \mathrm{mJy}$ & $190 \mathrm{deg}^{2} \mathrm{~h}^{-1}$ \\
\hline \hline
\end{tabular}

adjust the array configuration (remove a tile from the good list).

Together, the components listed above act in concert to enable a very flexible and robust $\mathrm{M} \& \mathrm{C}$ system. To date, the system has performed well, with the inherent flexibility and the volume of monitoring data serving as a great resource in commissioning and debugging the MWA. Partly because of the modular nature of the MWA, the M\&C system is inherently scalable to many hundreds of tiles and may provide a useful starting point for future arrays with large numbers of antennas.

\section{MWA PERFORMANCE}

For the MWA as described in this paper, the time (in seconds) needed to reach a point source sensitivity of $\sigma_{\mathrm{s}}$ (in Jy) is

$$
t=\left(\frac{2 k_{\mathrm{B}} T}{A_{\mathrm{eff}} N \epsilon_{\mathrm{c}}}\right)^{2} \frac{1}{\sigma_{\mathrm{s}}^{2} B n_{\mathrm{p}}},
$$

where $k_{\mathrm{B}}$ is the Boltzmann constant, $T=T_{\text {sky }}+T_{\text {rcv }}$ is the system temperature (in Kelvin), $A_{\text {eff }}$ is the effective area of each antenna tile (in $\mathrm{m}^{2}$ ), $N$ is the number of antenna tiles, $\varepsilon_{\mathrm{c}}$ is the correlator efficiency (dimensionless; assumed unity for the MWA), $B$ is the instantaneous bandwidth (in $\mathrm{MHz}$ ), and $n_{\mathrm{p}}$ is the number of polarisations. For the parameters given in Table 1 at $150 \mathrm{MHz}$, this equation reduces to $t \approx 8 \times$ $10^{4} / B \sigma_{\mathrm{s}}^{2} \mathrm{~s}$, for $\sigma_{\mathrm{s}}$ in $\mathrm{mJy}$.

Table 2 lists approximate derived point source and surface brightness sensitivities and survey speeds based on the equation above. The values listed in Table 2 are calculated assuming that the MWA's system temperature is dominated by sky noise. In practice, the imaging performance of the array will be affected by confusion for Stokes I images and the system temperature may be affected by subtle effects from short baselines. The remainder of this section discusses these issues, informed by our experience with the 32-tile test array data.

\subsection{Sky Noise Dominated Performance}

Here we describe how the performance of the 32-tile test array was verified to be sky noise dominated. The performance metric of a radio telescope that is directly measurable is the system equivalent flux density (SEFD), defined as $\mathrm{SEFD}=2 k_{\mathrm{B}} T / A_{\mathrm{eff}}$. For a sky noise dominated telescope, 


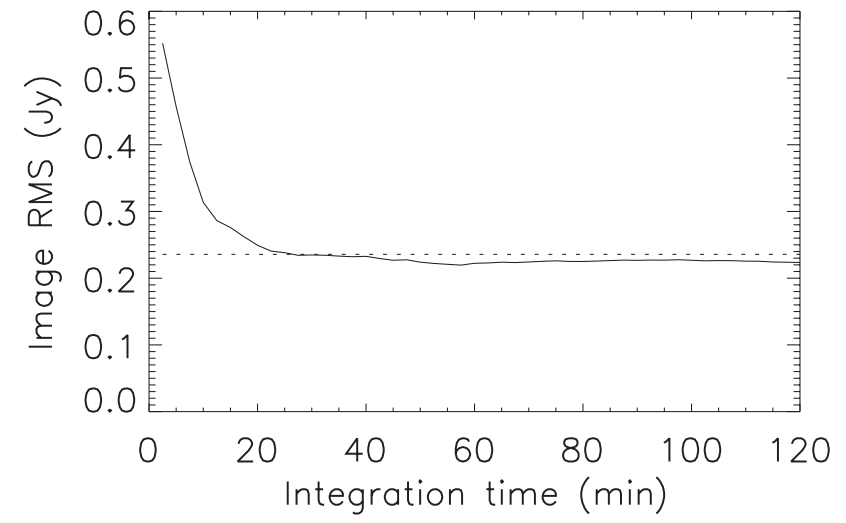

Figure 11. Measured RMS in a synthesised image as a function of the integration time (solid line). The dotted line indicates the expected classical confusion limit for the image.

the SEFD is determined by the average sky temperature seen by the antenna primary beam $\left(T_{\text {sky }}\right)$ and $A_{\text {eff }}$. For the MWA at $200 \mathrm{MHz}$ using the sky temperature from Table 1, this is approximately $22000\left(T_{\text {sky }} / 170 \mathrm{~K}\right) \mathrm{Jy}$.

Using calibrated visibilities with fine frequency and time resolution (where thermal noise dominates the correlated sky signal), the RMS noise in visibilities is a direct measure of the SEFD. Using this method, we determined the typical SEFD of the tiles based on calibrated visibilities to be approximately $20000 \mathrm{Jy}$ when the array is pointed at a cold patch of the sky well away from the Galactic plane, consistent with being sky noise limited.

\subsection{Confusion}

The confusion-limited Stokes I imaging performance of the 32-tile test array was verified by measuring the RMS variation in a multi-frequency synthesis image, for successively longer integrations on a field and a centre observing frequency of $155 \mathrm{MHz}$, and comparing the measurement to a calculation of the classical confusion limit.

To estimate the expected classical confusion in the image, we follow Condon (1974) and use the $6 \mathrm{C}$ survey at $151 \mathrm{MHz}$ (Hales, Baldwin, \& Warner 1988). We approximate the differential source counts below $1 \mathrm{Jy}$ as a power law given by $n(S)=3600 S^{-1.6} \mathrm{Jy}^{-1} \mathrm{sr}^{-1}$. The expected confusion level depends on the slope and normalisation of $n(S)$, the S/N cutoff $q$ for source identification, and the details of how the image was formed, in particular the size of the synthesised beam. Using a standard S/N cut-off of $q=5$ and the synthesised beam of the deconvolved image, the RMS confusion for the 32-tile test array was estimated to be $0.23 \mathrm{Jy}$.

The results of this test are shown in Figure 11. The RMS level in the image levels off as a function of integration time, as expected by classical confusion. The measured confusion floor is in good agreement with the estimated value. A noteworthy feature of the figure is the higher than expected RMS for short ( $<10 \mathrm{~min}$ ) integration times (compared to calcu- lations using the radiometer equation for Stokes I). This is due to the additional sidelobe confusion caused by the combined sidelobes of un-deconvolved confusing sources in the sky. The magnitude of the sidelobe confusion reduces with increased integration time because increased hour-angle coverage and filling of the $u v$ plane reduces the sidelobes of the synthesised beam.

Using the same parameters, the classical source confusion limit is estimated to be $\sim 6 \mathrm{mJy}$ at $150 \mathrm{MHz}$, assuming a synthesised beam full width at half maximum (FWHM) of 5 arcmin. It is worth noting that the Stokes I sky survey is the only key science goal (see next section) that will be confusion limited.

\section{MWA SCIENCE GOALS}

The MWA will be capable of a wide range of science investigations. These planned investigations are described in detail by J. Bowman et al. (2012). Here, we review the four key science themes that encompass the planned investigations and that have driven the design of the array. The key science themes for the array are (1) detection of fluctuations in the brightness temperature of the diffuse redshifted 21-cm line of neutral hydrogen from the EoR; (2) studies of Galactic and extragalactic processes based on a deep, confusion-limited survey of the full sky visible to the array; (3) time-domain astrophysics through exploration of the variable radio sky (transients); and (4) solar heliosphere and ionosphere imaging and characterisation via propagation effects on background radio sources.

Exploration of the Cosmic Dawn, the period that includes the formation of the first stars and galaxies in the early Universe, has been identified as an important area for new discoveries within the next decade. The MWA will be one of the first radio interferometers to attempt to detect redshifted $21-\mathrm{cm}$ line emission from neutral hydrogen gas in the intergalactic medium at the end of the Cosmic Dawn. The array has been designed to optimise its ability to detect brightness temperature fluctuations in the $21-\mathrm{cm}$ line emission during the reionisation epoch in the redshift range $6<z<$ 10. During the EoR, primordial neutral hydrogen begins to be ionised by the radiation from the first luminous sources. The MWA has sufficient thermal sensitivity to detect the presence of the large ionised voids that form during reionisation through measurements of the power spectrum and other statistical properties of the fluctuations to a significance level of $14 \sigma$ (Beardsley et al. 2012). In order to achieve this objective, the MWA will be a testbed to develop and demonstrate techniques to subtract the bright radio foregrounds that obscure the 21-cm background.

Radio emission from the Galaxy and from extragalactic sources is both a complicating foreground for EoR observations and an interesting scientific target that forms the second key science theme for the MWA. The MWA will be unique in its ability to conduct an arcminute resolution, confusionlimited survey of the full Southern Hemisphere sky below 
$10^{\circ}$ declination over the $80-300 \mathrm{MHz}$ frequency range. The survey will include the Galactic Centre and the Large and Small Magellanic Clouds. At the low observing frequencies of the MWA, non-thermal processes and Faraday rotation (and depolarisation) effects will be prominent. The MWA should be particularly well suited to identifying the missing population of old and faint supernova remnants (SNRs) in the Galaxy, closing the gap between the $\sim 300$ known SNRs and the expected 1 000-2 000, and thereby providing a critical measurement of the total energy budget of the interstellar medium. Additional experiments planned for the MWA target radio relics and clusters, the cosmic web, and Faraday tomography to probe magnetic fields. Cosmic-ray mapping may be also be possible along sight-lines where sufficiently dense $\mathrm{H}$ II regions have become optically thick in the MWA frequency band, blocking synchrotron emission from the Galaxy behind them.

With its high survey efficiency, as well as planned long integrations on EoR target fields, the MWA will enable sensitive transient and variable searches for both rare and faint events on timescales from seconds to days. The MWA will perform blind searches and target known transient sources, including low-mass stars and brown dwarfs, pulsars, X-ray binaries, and isolated neutron stars. At time resolution in the $\mu s-1$ s range, the voltage capture capability of the MWA will allow studies of pulsars at low frequencies and searches for fast transients (e.g. Wayth et al. 2012).

The final key science theme for the MWA encompasses the field of space weather, targeting multiple aspects of solar bursts as they travel from the surface of the Sun to the Earth. The primary science focuses on high-dynamic-range spectroscopic imaging to map the frequency, spatial, and time evolution of radio bursts occurring in the solar corona at heights of approximately 1-4 solar radii. Interplanetary scintillation will be used to constrain the density and turbulence of the interstellar wind in the inner heliosphere. The MWA may also enable measurements of the magnetic field of the heliosphere plasma if it proves possible to track changes in the polarisation angles of background sources. Lastly, the calibration solutions of the MWA will yield near-real-time corrections for ionospheric distortions, providing a new window into variability in the Earth's ionosphere.

\section{DISCUSSION}

The MWA will be the first of the SKA Precursors to come to completion. The MWA will also be the only low-frequency SKA Precursor and the most capable general-purpose lowfrequency radio telescope in the Southern Hemisphere, located on an extremely low-interference site at the MRO. The MWA is therefore a unique facility and highly complementary to ASKAP (also located at the MRO), with access to a similar FoV, but with ASKAP operating at higher frequencies (700-1800 MHz). This frequency diversity potentially allows simultaneous coverage of interesting astronomical phenomena over the frequency range $80-1800 \mathrm{MHz}$ from the MRO.
The MWA is also highly complementary to LOFAR, as the premier low-frequency radio telescope in the Northern Hemisphere, as well as the long wavelength array (LWA) (Taylor et al. 2012), providing complementary coverage of the whole sky and also longitudinal coverage important for observations of the Sun.

As one of a range of new facilities being brought online around the world now and in the future, many with an emphasis on high sensitivity and/or wide FoV survey science, a comparison of relevant metrics is useful. In particular, a prime metric of importance for new survey science programs is the survey speed (SS), defined as (Cordes 2010)

$$
\mathrm{SS}=\frac{\Omega_{\mathrm{FoV}}}{\tau},
$$

where $\Omega_{\mathrm{FoV}}$ is the solid angle subtended by the FoV of the telescope in question, and $\tau$ is the time required for an image of that FoV to reach a particular level of sensitivity. The wider the FoV of the primary array elements and the more primary elements in the array, the faster an array can survey a given region of the sky. ASKAP, the MWA, and LOFAR are well suited to survey science, as is the planned SKA. However, it needs to be noted that the angular resolution of the instrument is an important factor related to survey speed, driven by the configuration of the primary elements, impacting the confusion limit for wide-field surveys. These considerations are, in turn, driven by the survey science goals for any given instrument.

A comparison of the survey speed metric is provided in Figure 12, for various existing and future instruments.

Figure 12 shows that the MWA is highly competitive in terms of FoV on the sky, at an order of magnitude lower sensitivity than LOFAR, but of comparable sensitivity to the giant metre-wave radio telescope (GMRT). In producing this comparison, the low-frequency instruments are shown with corrections for spectral indices of -0.7 and -2.0 , corresponding to incoherent and coherent emission processes, respectively, in order to compare to the higher frequency instruments, which are all shown for an observing frequency of $1.4 \mathrm{GHz}$. This style of comparison follows Fender \& Bell (2011). Additionally, in Figure 12, the aperture array instruments are assumed to be pointed at zenith and a common sky temperature is assumed at low frequencies following Nijboer, Pandey-Pommier, \& de Bruyn (2009). The solid lines shown denote constant depth and constant volume figures of merit, arbitrarily normalised to the MWA point at $200 \mathrm{MHz}$.

The MWA is therefore very well suited to large-scale and repeated surveys of the sky between 80 and $300 \mathrm{MHz}$, down to the expected confusion limit. An expansion of the MWA at some future date to 256 tiles would move the MWA points in Figure 12 higher by a factor of 2 in sensitivity.

In its formal role as an SKA Precursor, the MWA feeds information to the international SKA project, primarily by informing activities such as the development of conceptual 


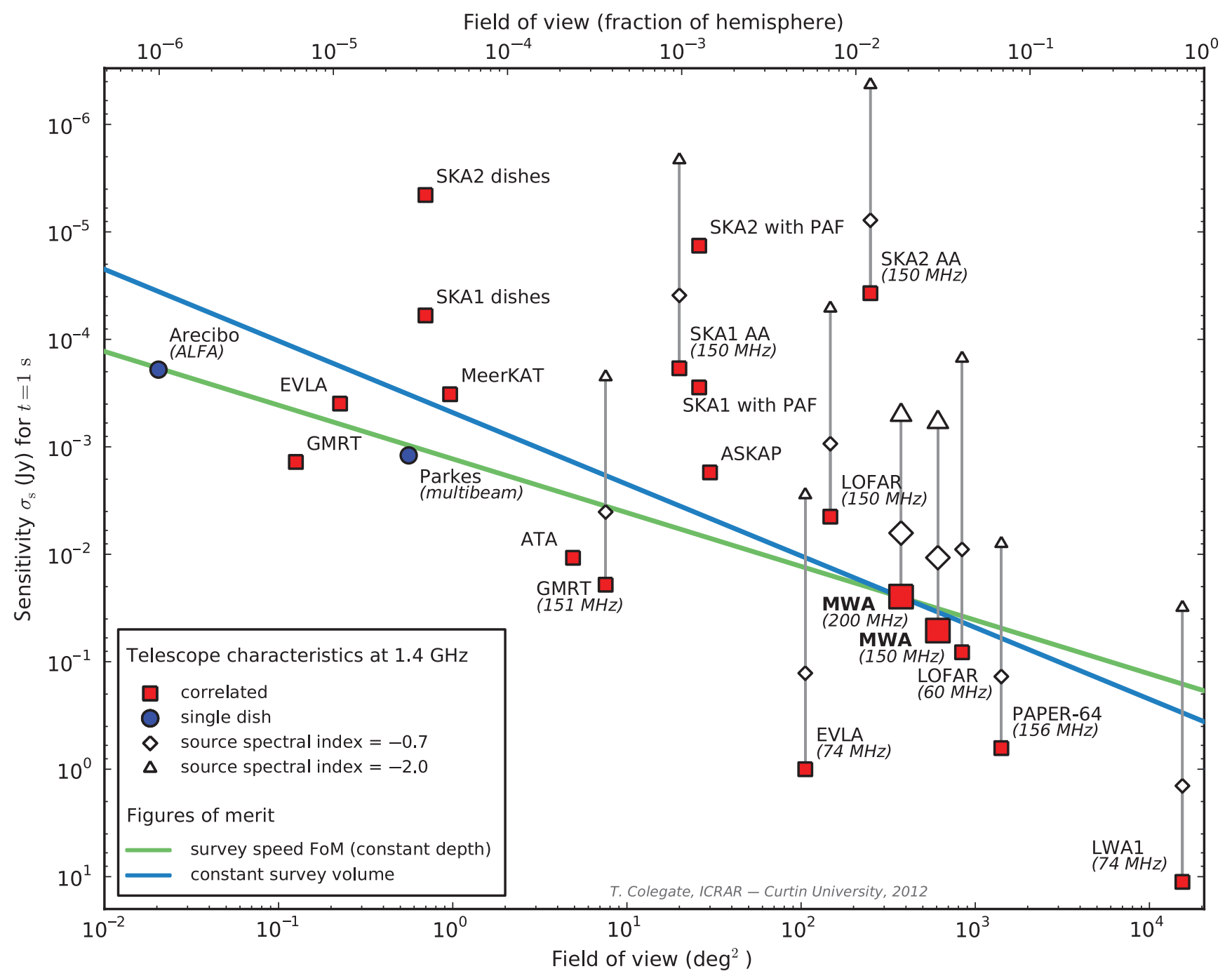

Figure 12. Comparison of the FoV and sensitivity for existing and future radio telescopes. Red squares denote interferometric telescopes. Blue circles denote single-dish telescopes. For the low-frequency instruments, spectral index corrections to $1.4 \mathrm{GHz}$ are indicated by the open diamonds and triangles, to allow comparison with higher frequency instruments. Note that in this figure, sensitivity increases to the top of the figure $\left(\sigma_{\mathrm{s}}\right.$ decreases). Figures of merit as discussed in the text are indicated by the blue and green lines.

design reviews for the low-frequency component of the SKA, and by reporting technical and scientific results into international SKA fora.

Important parts of the MWA design are based upon novel and new approaches to radio astronomy. For example, the use of aperture arrays to implement electronically steerable antennas with high flexibility and low cost is a pathfinder activity for the SKA, and the MWA and LOFAR are pioneering this technology on medium to large scales. While electronic steering with no moving parts has been used before, notably with the teepee-tee (TPT) array at Clark Lake (Erickson \& Fisher 1974) (also used at Nancay), such systems typically used phase rotation which is a narrow-band technique. The MWA instead uses switched delay lines with fine-grained steps, implemented using modern fabrication techniques to achieve the goals of low-cost, accurately steerable beams with a wide fractional bandwidth.
The choice of siting the MWA at the MRO, as part of a greenfield development in search of the best environments for low-frequency radio astronomy, clearly has massive benefits for low-frequency science, but comes with significant challenges. The practical challenges attendant to the construction of highly complex systems, in locations well away from the traditional centres of support for radio astronomy, are challenges that the SKA will have to face on an even larger scale. Lessons learnt from the MWA have a deep relevance to how the SKA project could proceed.

The MWA is expected to have an approximate 5-year lifetime, in order to achieve its science goals. Beyond the operational lifetime of the facility, we note that the technologies that have gone into the MWA analog, digital and signal processing systems will be of the order of a decade old and it is likely that new, more efficient, more powerful, and less power-intensive hardware will be available for the 
same task. It is possible, therefore, to imagine a future reuse of the MWA infrastructure (power and fibre reticulation, capacity in CPF, and data transport to the Pawsey Centre) that could support a new and much more capable successor to the MWA, on a 5-10 year timescale. This scenario highlights the enormous value created in building highly capable infrastructure at a remote and, from an RFI point of view, pristine site. Such a scenario is supported by the fact that the fielded MWA equipment in tiles, beamformers, and receivers are all highly portable and can be easily decommissioned and replaced. Thus, the MWA consortium recognises a long-term future for low-frequency radio astronomy at the MRO, through the MWA initially and then, perhaps, subsequent generations of instruments that re-use the underlying infrastructure.

An immediate possibility that takes advantage of the MWA infrastructure is for the facility to host some of the required prototyping activities for the low-frequency sparse aperture array component of the SKA. As the MRO is the internationally endorsed host site for the low-frequency SKA in Phases 1 and 2, an immediate advantage to the SKA project could be derived through use of the MWA infrastructure in testing and verifying the performance of prototype SKA hardware, through the international SKA Aperture Array Verification Programme (AAVP).

\section{ACKNOWLEDGMENTS}

We acknowledge the Wajarri Yamatji people as the traditional owners of the Observatory site. Support for the MWA comes from the U.S. National Science Foundation (grants AST CAREER0847753, AST-0457585, AST-0908884, and PHY-0835713), the Australian Research Council (LIEF grants LE0775621 and LE0882938), the U.S. Air Force Office of Scientific Research (grant FA9550-0510247), the Centre for All-sky Astrophysics (an Australian Research Council Centre of Excellence funded by grant CE110001020), New Zealand Ministry of Economic Development (grant MED-E1799), an IBM Shared University Research Grant (via VUW and Curtin), the Smithsonian Astrophysical Observatory, the MIT School of Science, the Raman Research Institute, the Australian National University, the Victoria University of Wellington, the Australian Federal government via the National Collaborative Research Infrastructure Strategy, Education Investment Fund and the Australia-India Strategic Research Fund and Astronomy Australia Limited, under contract to Curtin University, the iVEC Petabyte Data Store, the Initiative in Innovative Computing and NVIDIA sponsored CUDA Center for Excellence at Harvard, and the International Centre for Radio Astronomy Research, a Joint Venture of Curtin University and The University of Western Australia, funded by the Western Australian State government.

\section{REFERENCES}

Beardsley, A. et al. 2012, MNRAS, 425, 1781

Bernardi, G. et al. 2011, MNRAS, 413, 411

Bernardi, G. et al. 2012, ApJ submitted

Bhatnagar, S., Cornwell, T. J., Golap, K., \& Uson, J. M. 2008, A\&A, 487,419
Bowman, J. 2012, PASA submitted

Bowman, J. D., \& Rogers, A. E. E. 2010, Natur, 468, 796

Bracewell, R. N. 1984, in Indirect Imaging, ed. Roberts, J. A. (Cambridge: Cambridge University Press), 177

Carilli, C., \& Rawlings, S. 2012, NewAR, 48, 979

Clark, M. A., LaPlante, P. C., \& Greenhill, L. J. 2012, arXiv: 1107.4264

Condon, J. J. 1974, ApJ, 188, 279

Cordes, J. M. 2007, SKA Memo \#109, Survey Metrics, www. skatelescope.org/pages/page_memos.htm

Cornwell, T. J., Golap, K., \& Bhatnagar, S. 2005, in ASP Conf. Ser. Vol. 347, Astronomical Data Analysis Software and Systems XIV (San Francisco, CA: ASP), 86

Cornwell, T. J., \& Perley, R. A. 1992, A\&A, 261, 353

Cornwell, T. J., Voronkov, M. A., \& Humphreys, B. 2012, Proc. SPIE, 8500, arXiv: 1207.5681

Deller, A. T., Tingay, S. J., Bailes, M., \& West, C. 2007, PASP, 119 , 318

Deller, A. T. et al. 2011, PASP, 123, 275

Dewdney, P., et al. 2010, SKA Memo \#130, SKA Phase 1: Preliminary System Description, www.skatelescope.org/pages/page memos.htm

Edgar, R. G. et al. 2010, Comput. Phys. Commun., 181, 1707

Erickson, W. C., \& Fisher, J. R. 1974, Radio Sci, 9, 387

Erickson, W. C., Perley, R. A., Flatters, C., \& Kassim, N. E. 2001, A\&A, 366, 1071

Fender, R., \& Bell, M. E. 2011, BASI, 39, 315

Gorski, K. M., et al. 2005, ApJ, 622, 759

Hales, S. E. G., Baldwin, J. E., \& Warner, P. J. 1988, MNRAS, 234, 919

Hamaker, J. P. 2000, A\&ASS, 143, 515

Johnston, S., et al. 2007, PASA, 24, 174

Johnston, S., et al. 2008, ExA, 22, 151

Lonsdale, C. J., et al. 2009, Proc. IEEE, 97, 1497

McKinley, B. et al. 2012, PJ, in press (arXiv:1211.1433)

Mitchell, D. A., Wayth, R. B., Bernardi, G., Greenhill, L. J., \& Ord, S. M. 2012, JAI, in press

Mitchell, D. A., et al. 2008, IEEE J. Sel. Top. Signal Process., 2, 1993

Mitchell, D. A., et al. 2010, PoS(RFI2010)016

Morales, M. F., \& Matejek, M. 2009, MNRAS, 400, 1814

Myers, S. T., et al. 2003, ApJ, 591, 575

Nieuwpoort, R. V. V., \& Romein, J. W. 2009, in ACM International Conference on Supercomputing (New York: ACM), 440

Nijboer, R. J., Pandey-Pommier, M., \& de Bruyn, A. G. 2009, SKA Memo \#113, LOFAR Imaging Capabilities and System Sensitivity, www.skatelescope.org/pages/page_memos.htm

Noordam, J. E. 2004, Proc. SPIE, 5489, 817

Oberoi, D., et al. 2011, ApJ, 728, L27

Ord, S. M., et al. 2009, Proceedings of Astronomical Data Analysis Software and Systems XVIII, ASP conf. ser.411 (eds. Bohlender, David A., Durand, Daniel \& Dowler, Patrick), 127

Ord, S. M., et al. 2010, PASP, 122, 1353

Perley, R. A. 1999, in ASP Conf. Ser. Vol. 180, Synthesis Imaging in Radio Astronomy II, ed. G. B. Taylor, C. L. Carilli, \& R. A. Perley (San Francisco, CA: ASP), 383

Pindor, B., Wyithe, J. S. B., Mitchell, D. A., Ord, S. M., Wayth, R. B., \& Greenhill, L. J. 2011, PASA, 28, 46

Smirnov, O. M. 2011, A\&A, 527

Sullivan, I. et al. 2012, ApJ, 729, 17 
Taylor, G. B., et al. 2012, JAI, Vol \#1, 150004

Thompson, A. R., Moran, J. M., \& Swenson, G. W. Jr. 2001, Interferometry and Synthesis in Radio Astronomy (2nd edn; New York: Wiley)
Wayth, R. B., et al. 2012, ApJ, 753, L36

Wells, D. C., Greisen, E. W., \& Harten, R. H. 1981, A\&AS, 44, 363

Wicenec, A., \& Knudstrup, J. 2007, Msngr, 129, 27

Williams, C. L., et al. 2012, ApJ, 755, 47 